\title{
Parameterized Complexity of $k$-Anonymity: Hardness and Tractability
}

\author{
Stefano Beretta* $^{*} \quad$ Paola Bonizzoni ${ }^{\dagger} \quad$ Gianluca Della Vedova ${ }^{\ddagger} \quad$ Riccardo Dondi $^{\S}$ \\ Yuri Pirola
}

October 26, 2018

\begin{abstract}
The problem of publishing personal data without giving up privacy is becoming increasingly important. A clean formalization that has been recently proposed is the $k$-anonymity, where the rows of a table are partitioned in clusters of size at least $k$ and all rows in a cluster become the same tuple, after the suppression of some entries. The natural optimization problem, where the goal is to minimize the number of suppressed entries, is hard even when the stored values are over a binary alphabet and as well as on a table consists of a bounded number of columns. In this paper we study how the complexity of the problem is influenced by different parameters. First we show that the problem is W[1]-hard when parameterized by the value of the solution (and $k$ ). Then we exhibit a fixed-parameter algorithm when the problem is parameterized by the number of columns and the maximum number of different values in any column. Finally, we prove that $k$-anonymity is still APX-hard even when restricting to instances with 3 columns and $k=3$.
\end{abstract}

\section{Introduction}

In epidemic studies the analysis of large amounts of personal data is essential. At the same time the dissemination of the results of those studies, even in a compact and summarized form, can provide some information that can be exploited to identify the row pertaining to a certain individual. For instance, ZIP code, gender and date of birth can uniquely identify $87 \%$ of individuals in the U.S. [18]. Therefore when managing personal data it is of the utmost importance to effectively protect individuals' privacy.

One approach to deal with such problem is the $k$-anonymity model [16, 18, 15, 12]. Each row of a given table represents all data regarding a certain individual. Then different rows are clustered together, and some entries of the rows in each cluster are suppressed (i.e. they are replaced with $\mathrm{a} *$ ) so that each cluster consists of at least $k$ identical rows. Therefore each row $r$ in the resulting table is clustered with at least other $k-1$ rows identical to $r$, hence the resulting

\footnotetext{
*DISCo, Università degli Studi di Milano-Bicocca, Milano - Italy

${ }^{\dagger}$ DISCo, Università degli Studi di Milano-Bicocca, Milano - Italy

${ }^{\ddagger}$ Dipartimento di Statistica, Università degli Studi di Milano-Bicocca, Milano - Italy

$\S$ Dipartimento di Scienze dei Linguaggi, Università degli Studi di Bergamo, Bergamo - Italy

${ }^{\top}$ DISCo, Università degli Studi di Milano-Bicocca, Milano - Italy
} 
data do not allow to identify any individual. While such formulation is not really sophisticated and has some practical limitations, it is definitely interesting from a theoretical point of view, as witnessed by the rich literature available. We will focus on separating the cases that can be solved efficiently from those that are intractable, therefore hinting at which strategies are likely or not going to be successfully employed when studying more sophisticated formalizations. Notice that different formulations of the problem have also been proposed [1, for example allowing the generalization of entry values, that is an entry value can be replaced with a less specific value [3, or considering a notion of proximity among values [10].

A parsimonious principle leads to the optimization problem where we want to minimize the number of entries in the table to be suppressed. The $k$-anonymity problem is known to be APX-hard even when the matrix entries are over a binary alphabet and $k=3$ [6], as well as when the matrix has 8 columns and $k=4$ (this time on arbitrary alphabets) [6]. Furthermore, a polynomial-time $O(k)$-approximation algorithm on arbitrary input alphabet, as well as approximation algorithms for restricted cases are known [2]. Recently, two polynomial-time approximation algorithms with factor $O(\log k)$ have been independently proposed [14, 11].

In this paper we investigate the parameterized complexity [8, 13] of the problem, unveiling how different parameters are involved in the complexity of the problem. A first systematic study of the parameterized complexity of the $k$-anonymity problem has been proposed in [7]. Here, we follow the same direction, showing that the problem is W[1]-hard when parameterized by the size of the solution and $k$, and we provide a fixed-parameter algorithm, when the problem is parameterized by the number of columns and the maximum number of different values in any column. These problems were left open in [7].

In Table 1 we report the status of the parameterized complexity of the $k$-anonymity problem, where in bold we have emphasized the new results presented in this paper. We recall that a problem $P$ parameterized by a set $Y$ of parameters is in the class FPT [8] if it admits an exact algorithm with complexity $f(Y) n^{O(1)}$, where $f$ is an arbitrary function, and $n$ is the size of the input problem, while it is $\mathrm{W}[\mathrm{i}]$-hard [8], for some $1 \leq i \leq p$ if it is unlikely to be fixed-parameter tractable. We recall that XP $[8]$ is a superclass of all sets $\mathrm{W}[p]$. Moreover, proving that a problem $\Pi$ with parameter set $S$ is NP-hard when all parameters in $S$ are some constants, implies that $(\Pi, S) \notin \mathrm{XP}$ unless $\mathrm{P}=\mathrm{NP}$.

\begin{tabular}{|c|c|c|c|c|}
\hline & - & $k$ & $e$ & $k, e$ \\
\hline- & NP-hard [12] & $\notin X P[6,2]$ & W[1]-hard new & W[1]-hard new \\
\hline$|\Sigma|$ & $\notin X P[\underline{6}$ & $\notin X P[\underline{6}$ & ??? & ??? \\
\hline$m$ & $\notin X P$ for $m \geq 8[\underline{6}$ & $\notin X P$ for $m \geq 8, k \geq 4[6]$ & FPT [7] & FPT [7] \\
\hline$n$ & FPT [7] & FPT [7] & FPT [7] & FPT [7] \\
\hline$|\Sigma|, m$ & FPT new & FPT [7] & FPT [7] & FPT [7] \\
\hline$|\Sigma|, n$ & FPT 7 & FPT 7 & FPT [7] & FPT 7 \\
\hline
\end{tabular}

Table 1: Summary of the parameterized complexity status of the $k$-anonymity problem; $|\Sigma|$ represents the maximum number of different values in a column, $m$ represents the number of columns, $n$ represents the number of rows, $k$ represents the minimum size of a cluster, $e$ represents the size of the solution.

The rest of the paper is organized as follows. In Section 2 we introduce some preliminary definition and we give the formal definition of the $k$-anonymity problem. In Section 3 we show that the $k$-anonymity is W[1]-hard. In Section 4 we give a fixed parameter algorithm, when the problem is parameterized by the size of the alphabet and the number of columns. Finally, in 
Section 5 we show that the 3 -anonymity problem is APX-hard, even when the rows have length bounded by 3 .

\section{Preliminary Definitions}

Let us introduce some preliminary definitions that will be used in the rest of the paper. Given a graph $G=(V, E)$, and $V^{\prime} \subseteq V$, the subgraph induced by $V^{\prime}$ is denoted by $G\left[V^{\prime}\right]=\left(V^{\prime}, E^{\prime}\right)$, where $E^{\prime}=E \cap\left(V^{\prime} \times V^{\prime}\right)$. A graph $G=(V, E)$ is cubic when each vertex in $V$ has degree three.

Given an alphabet $\Sigma$, a row $r$ is a vector of elements taken from the set $\Sigma$, and the $j$-th element of $r$ is denoted by $r[j]$. Notice that it is equivalent to consider a row as a vector over $\Sigma$ or as a string over alphabet $\Sigma$. Let $r_{1}, r_{2}$ be two equal-length rows. Then $H\left(r_{1}, r_{2}\right)$ is the Hamming distance of $r_{1}$ and $r_{2}$, i.e. $\left|\left\{i: r_{1}[i] \neq r_{2}[i]\right\}\right|$. Let $R$ be a set of $l$ rows, then a clustering of $R$ is a partition $\Pi=\left(P_{1}, \ldots, P_{t}\right)$ of $R$. Given a clustering $\Pi=\left(P_{1}, \ldots, P_{t}\right)$ of $R$, we define the cost of the row $r$ belonging to a set $P_{i}$ of $\Pi$ as $c_{\Pi}(r)=\left|\left\{j: \exists r_{1}, r_{2} \in P_{i}, r_{1}[j] \neq r_{2}[j]\right\}\right|$, that is the number of entries of $r$ that have to be suppressed so that all rows in $P_{i}$ are identical. Similarly we define the cost of a set $P_{i}$, denoted by $c_{\Pi}\left(P_{i}\right)$, as $\left|P_{i} \|\left\{j: \exists r_{1}, r_{2} \in P_{i}, r_{1}[j] \neq r_{2}[j]\right\}\right|$. The cost of $\Pi$, denoted by $c(\Pi)$, is defined as $\sum_{P_{i} \in \Pi} c\left(P_{i}\right)$. Given a set $S \subseteq R$ and a clustering $\Pi$ of $R$, the cost induced by $\Pi$ in set $S$ is $c_{\Pi}(S)=\sum_{r \in S} c_{\Pi}(r)$. Notice that, given a clustering $\Pi=\left(P_{1}, \ldots, P_{t}\right)$ of $R$, the quantity $\left|P_{i}\right| \max _{r_{1}, r_{2} \in P_{i}}\left\{H\left(r_{1}, r_{2}\right)\right\}$ is a lower bound for $c\left(P_{i}\right)$, since all the positions for which $r_{1}$ and $r_{2}$ differ will be deleted in each row of $P_{i}$. We are now able to formally define the $k$-Anonymity Problem ( $k$-AP).

Problem 1. $k-A P$.

Input: a set $R$ of equal lenght rows over an alphabet $\Sigma_{R}$.

Output: a clustering $\Pi=\left(P_{1}, \ldots, P_{t}\right)$ of $R$ such that for each set $P_{i},\left|P_{i}\right| \geq k$ and $c(\Pi)$ is minimum.

In what follows, given a set $S$ of parameters, we denote by $\langle S\rangle$-AP the $k$-AP problem parameterized by $S$, thus omitting $k$. We will consider the following parameters: $m$ is the number of columns of the rows in $R ; n$ is the number of rows in $R ;|\Sigma|$ is the maximum number of different values in any column of the table; $k$ is the minimum size of a cluster; $e$ is the maximum number of entries that can be suppressed.

Let $\Pi=\left(P_{1}, \ldots, P_{z}\right)$ be a solution of the $k$-AP problem. Notice that a suppression at position $j$ of a row $r$ is represented replacing the symbol $r[j]$ with a $*$. Given a set $P_{j}$ of $\Pi$, some entries of the rows clustered in $P_{j}$ are suppressed, so that the resulting rows are all identical to a vector $r$ over alphabet $\Sigma_{R} \cup\{*\}$; such a vector is the resolution vector associated with $P_{j}$. Given a resolution vector $r$, we define $\operatorname{del}(r)$ as the number of entries suppressed in $r$, that is $\operatorname{del}(r)=|\{j: r[j]=*\}|$. Given a resolution vector $r$ and a row $r_{i} \in R$, we say that $r$ is compatible with row $r_{i}$ iff $r[j] \neq r_{i}[j]$ implies $r[j]=*$. Given a row $r_{i}$ of $R$ and a set of resolution vectors $S^{\prime}$, we define the set $\operatorname{comp}\left(r_{i}, S^{\prime}\right)=\left\{r \in S^{\prime}: r\right.$ is compatible with $\left.r_{i}\right\}$.

Given a set $R$ of rows, we define a group of rows of $R$ as a maximal set of identical rows. Given a group $g$, the representative row of $g$, denoted by $r(g)$, is any row of $g$, while $s(g)$ is the number of rows in $g$ and $\operatorname{exc}(g)=\max \{0, s(g)-k\}$. A set $R$ of rows can be partitioned in groups of identical rows in polynomial time [7], therefore we can compute in polynomial time whether a set $R$ of rows is $k$-anonymous, i.e. $R$ can be partioned into groups of size at least $k$. If this is not possible, then observe that at least $k$ entries of $R$ must be suppressed to get a solution of 
the $k$-AP problem, that is $e \geq k$. Hence $\langle e\rangle$-AP is in FPT iff $\langle e, k\rangle$-AP is in FPT. Consequently our parameterized reduction [8, 13] will show the fixed-parameter intractability of $\langle e\rangle$-AP and $\langle e, k\rangle$-AP.

\section{$3\langle e\rangle$ - $\mathbf{A P}$ and $\langle e, k\rangle$-AP are $\mathbf{W}[1]$-hard}

We show that $\langle e\rangle$-AP and $\langle e, k\rangle$-AP are W[1]-hard. Given an set $R$ of equal length rows, $\langle e\rangle$-AP and $\langle e, k\rangle$-AP ask if there exists a clustering $\Pi=\left(P_{1}, \ldots, P_{t}\right)$ of $\mathrm{R}$ such that $\left|P_{i}\right| \geq k$ for each set $P_{i}$, and $c(\Pi) \leq e$. We present a parameter preserving reduction from the $h$-Clique problem, which is known to be $\mathrm{W}[1]$-hard [9], to the $\langle e\rangle$-AP problem. Given a graph $G=(V, E)$, an $h$-clique is a set $V^{\prime} \subseteq V$ where each pair of vertices in $V^{\prime}$ are connected by an edge of $G$, and $\left|V^{\prime}\right|=h$. The $h$-Clique problem asks for a subset $V^{\prime}$ of the vertices of a given graph $G$ inducing an $h$-clique in $G$.

Clearly the vertices of a $h$-clique are connected by $\left(\begin{array}{l}h \\ 2\end{array}\right)$ edges. Given a graph $G=(V, E)$, we use $m_{G}$ and $n_{G}$ to denote respectively the number of edges and of vertices of $G$. We construct the instance $R$ of $\langle e\rangle$-AP associated with $G$. First, let us define $k=2 h^{2}$. The set $R$ consists of $(k+1) m_{G}+\left(k-\left(\begin{array}{c}h \\ 2\end{array}\right)\right)$ rows and $2 h+n_{G}$ columns over alphabet $\Sigma_{R}=\{0,1\} \cup\left\{\sigma_{i, j}:\left(v_{i}, v_{j}\right) \in E\right\}$. More precisely, for each edge $e(i, j)=\left(v_{i}, v_{j}\right)$ in $E$, there is a group $R(i, j)$ of $k+1$ identical rows $r_{x}(i, j), 1 \leq x \leq k+1$, where

- $r_{x}(i, j)[l]=\sigma_{i, j}$, for $1 \leq l \leq 2 h$;

- $r_{x}(i, j)[2 h+i]=1, r_{x}(i, j)[2 h+j]=1$;

- $r_{x}(i, j)[2 h+l]=0$, for $l \neq i, j$ and $1 \leq l \leq n$.

Moreover, $R$ also contains a group $R_{0}$ made of $k-\left(\begin{array}{c}h \\ 2\end{array}\right)$ identical rows equal to $0^{2 h+n_{G}}$.

Lemma 1. Let $R$ be the instance of $\langle e\rangle-A P$ associated with $G$ and consider two rows $r, r_{x}(i, j)$ of $R$, such that $r \in R_{0}$ and $r_{x}(i, j) \in R(i, j)$. Then, $r[t] \neq r_{x}(i, j)[t]$, for each $1 \leq t \leq 2 h$.

Lemma 2. Let $G=(V, E)$ be a graph, let $V^{\prime}$ be a h-clique of $G$ and let $R$ be the instance of $\langle e\rangle-A P$ associated with $G$. Then we can compute in polynomial time a solution $\Pi$ of $\langle e\rangle$-AP over instance $R$ with cost at most $6 h^{3}$.

Lemma 3. Let $G=(V, E)$ be an instance of $h$-Clique, let $R$ be the instance of $\langle e\rangle$-AP associated with $G$ and let $\Pi$ be a solution of $\langle e\rangle-A P$ over instance $R$ with cost at most $6 h^{3}$. Then we can compute in polynomial time a h-clique $V^{\prime}$ of $G$.

Proof. First we will prove that $\Pi$ must have a set $R_{0}^{\prime} \supset R_{0}$. Assume to the contrary that in $\Pi$ there are two sets $A, B$ containing at least a row of $R_{0}$. Notice that $\left|R_{0}\right|<k$ while $|A|,|B| \geq k$. Moreover, by Lemma 1, all rows in $A$ or $B$ must have suppressed the first $2 h$ entries, which results in at least $4 h k>6 h^{3}$ suppressions, contradicting the assumption on the cost of the solution. Hence, $R_{0}$ is properly contained in a set $R_{0}^{\prime}$ of $\Pi$, as $\left|R_{0}\right|<k$. Moreover, let $r^{\prime}$ be a row of $R_{0}^{\prime} \backslash R_{0}$ and let $r$ be a row of $\in R_{0}$. By Lemma $1 r^{\prime}[t] \neq r[t]$ for each column $t, 1 \leq t \leq 2 h$, therefore all entries in the first $2 h$ columns of each row in $R_{0}^{\prime}$ must be suppressed.

Now, let us prove that, for each set $R(i, j)$ of $R$, there exists a set $R^{\prime}(i, j)$ of $\Pi$ such that $R^{\prime}(i, j) \subseteq R(i, j)$. Assume to the contrary that no such set $R^{\prime}(i, j)$ exists, for a given $R(i, j)$. 
Then either $R(i, j) \subseteq R_{0}^{\prime}$ or there exists a row of $R(i, j)$ clustered together with a row of $R(x, y)$ in $\Pi$, with $(x, y) \neq(i, j)$. In the first case, that is $R(i, j) \subseteq R_{0}^{\prime},\left|R_{0}^{\prime}\right| \geq 2 k+1-\left(\begin{array}{c}h \\ 2\end{array}\right)$, by construction all entries of the first $2 h$ columns of the rows in $R_{0}^{\prime}$ must be suppressed, resulting in at least $2 h\left(4 h^{2}-\left(\begin{array}{l}h \\ 2\end{array}\right)\right)>6 h^{3}$ suppressions and thus contradicting the assumption on the cost of the solution. Consider now the second case, that is there is a set $A$ in $\Pi$ containing at least a row of two different sets $R(i, j)$ and $R(x, y)$ of $R$. Observe that given $r^{\prime} \in R_{0}^{\prime} \backslash R_{0}$ and $r \in R_{0}, r$ and $r^{\prime}$ differ in the first $2 h$ columns. Thus the entries of the first $2 h$ columns of the rows of $R_{0}^{\prime}$ must be suppressed, resulting in at least $4 h k>6 h^{3}$ suppressed entries and thus contradicting the assumption on the cost of the solution. Hence, for each set $R(i, j)$ of $R$, there exists a set $R^{\prime}(i, j)$ of $\Pi$ such that $R^{\prime}(i, j) \subseteq R(i, j)$.

By our previous arguments we can assume that $\Pi$ consists of the clusters $R_{0}^{\prime}$ and $R^{\prime}(i, j)$, for each $R(i, j) \in R$, and that $|R(i, j)|-1 \leq\left|R^{\prime}(i, j)\right| \leq|R(i, j)|$. Notice that only $R_{0}^{\prime}$ can contain some suppressed entries. Also $\left|R_{0}^{\prime}\right|=k$, for otherwise we can improve the cost of $\Pi$ by moving a row in $R(i, j) \cap R_{0}^{\prime}$ from $R_{0}^{\prime}$ to $R^{\prime}(i, j)$. Now let $E^{\prime}$ be the set of edges $\left(v_{i}, v_{j}\right)$ of $G$ such that a row of $R(i, j)$ is in $R_{0}^{\prime}$ and let $V^{\prime}$ be the set of vertices incident on at least an edge in $E^{\prime}$. Then we can show that $G\left[V^{\prime}\right]$ is a $h$-clique. Notice that the entries in the first $2 h$ columns of $R_{0}^{\prime}$ must be suppressed, as well as all columns with index $2 h+l$ such that $v_{l} \in V^{\prime}$, since in those columns all rows in $R_{0}$ have value 0 while some row in $R_{0}^{\prime} \backslash R_{0}$ have value 1 . An immediate consequence is that the overall number of suppressed entries is at least $2 h k+k\left|V^{\prime}\right|$. Since, by hypothesis, the number of suppressed entries is at most $6 h^{3}=3 k h$, then $\left|V^{\prime}\right| \leq h$. Notice that, since $\left|R_{0}\right|=k-\left(\begin{array}{l}h \\ 2\end{array}\right)$ and $\left|R_{0}^{\prime}\right|=k$, then $R_{0}^{\prime} \backslash R_{0}$ contains exactly $\left(\begin{array}{c}h \\ 2\end{array}\right)$ distinct rows corresponding to edges in $E^{\prime}$ incident on $V^{\prime}$ vertices. Hence $V^{\prime}$ induces a $h$-clique in $G$.

From Lemma 2 and 3 , our reduction is parameter preserving, therefore $\langle e\rangle$-AP and $\langle e, k\rangle$-AP are W[1]-hard.

\section{An FPT algorithm for $\langle|\Sigma|, m\rangle$-AP}

In this section we present a fixed-parameter algorithm for the $\langle|\Sigma|, m\rangle$-AP problem, that is the instance of the AP problem, where the number $m$ of columns and the maximum number $|\Sigma|$ of different values in any column are two parameters. Notice that $k$-AP parameterized by exactly one of $|\Sigma|$ or $m$ is not in FPT, as $k$-AP is APX-hard (hence NP-hard) even when one of $|\Sigma|$ or $m$ is a constant [6].

Before giving the details of the algorithm, let us first introduce some preliminary definitions. Let $R$ be an instance of $\langle|\Sigma|, m\rangle$-AP, and for each column of $R$ with index $j, 1 \leq j \leq m$, let $\Sigma_{j}$ be the set of different values that the rows of $R$ have in column $j$. Notice that $\left|\Sigma_{j}\right| \leq|\Sigma|$, for each $1 \leq j \leq m$. Let $\Sigma_{j}^{*}=\Sigma_{j} \cup\{*\}$ and $\Sigma^{*}=\Sigma \cup\{*\}$. Assume $\Pi=\left\{P_{1}, \cdots, P_{z}\right\}$ is a feasible solution of $\langle|\Sigma|, m\rangle$-AP over instance $R$. The set $S^{\prime}$ consisting of a resolution vector for each set $P_{i} \in \Pi$ is called candidate set for solution $\langle|\Sigma|, m\rangle$-AP. Let $S$ be the set of possible rows of length $m$ and having value over alphabet $\Sigma_{j}^{*}$ for the position $j, 1 \leq j \leq m$, then $|S|$ is bounded by $\left|\Sigma^{*}\right|^{m}$. Given a candidate set $S^{\prime}$, notice that $S^{\prime} \subseteq S$ and that each row $r \in R$ must compatible with at least one resolution vector in $S^{\prime}$.

Given a row $r$ and the set $S^{\prime}$ of resolution vectors, recall that we denote by $\operatorname{Comp}\left(r, S^{\prime}\right)$ the set of resolution vectors of $S^{\prime}$ compatible with $r$. Moreover, given a resolution vector $r^{\prime} \in S^{\prime}$, we denote by $\operatorname{del}\left(r^{\prime}\right)$ the number of suppressions in $r^{\prime}$. For each row $r \in R$ we define its weight as $w(r)=\max _{r_{x} \in \operatorname{Comp}\left(r, S^{\prime}\right)}\left\{m-\operatorname{del}\left(r_{x}\right)\right\}$. Notice that $w(r)=m$ whenever $r$ is compatible with a 


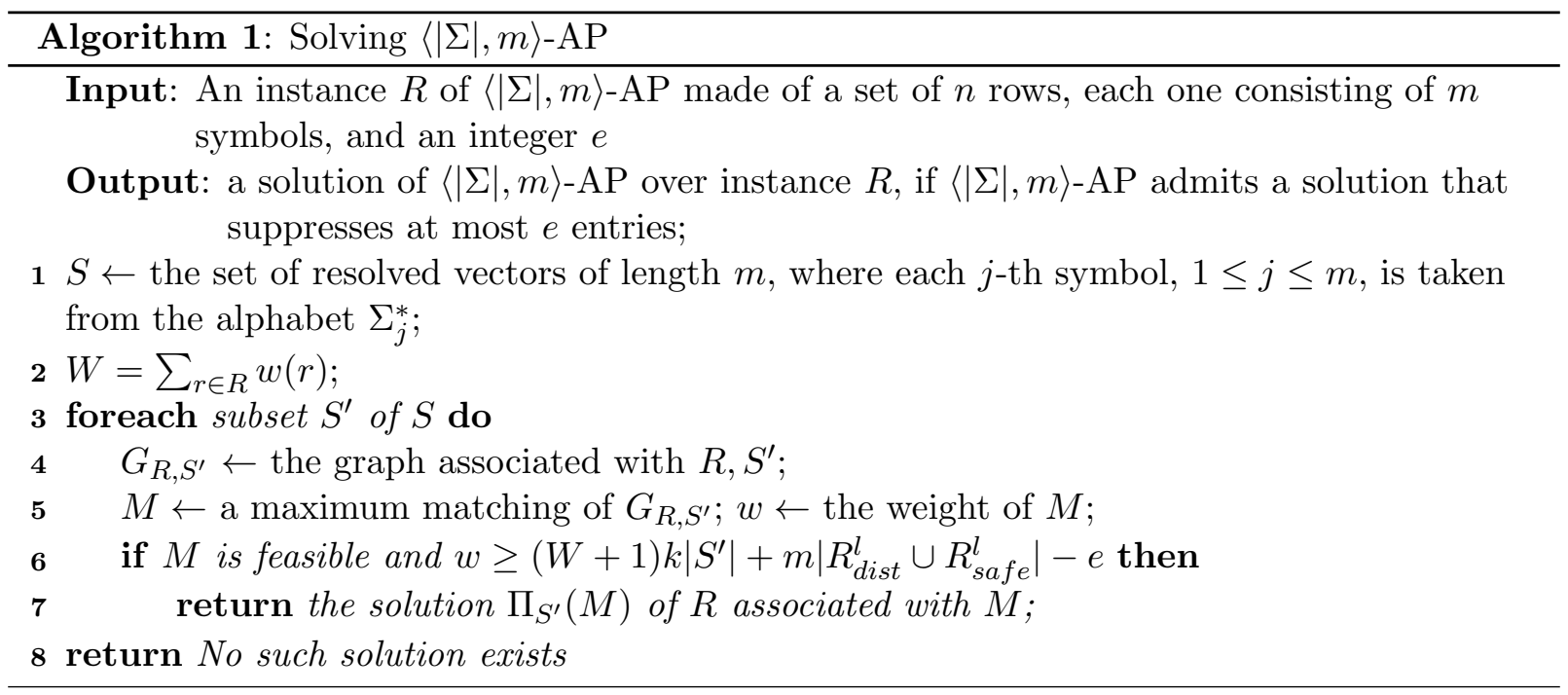

row without suppressions. Informally, the weight of a row is equal to the maximum number of its entries that might be preserved in a solution where $S^{\prime}$ is the set of resolution vectors. Finally, we define $W=\sum_{r \in R} w(r)$ and $w^{\prime}\left(r_{x}\right)=W+m-\operatorname{del}\left(r_{x}\right)+1$ for each row $r_{x} \in S^{\prime}$. Notice that $w^{\prime}\left(r_{x}\right) \geq \sum_{r \in R} w(r)$, for each $r_{x} \in R$. The weights defined above will be used later in Section 4.1 to define the weight function $w_{h}$.

Let us first describe the general idea of the algorithm. Given a candidate set $S^{\prime}$, the algorithm computes an optimal solution $\Pi_{S^{\prime}}$ associated with a candidate set $S^{\prime} \subseteq S$ (see Algorithm 1). The algorithm consists of two main phases. In the first phase (Section 4.1), given the set $R$ of input rows and the candidate set $S^{\prime}$, the algorithm builds a weighted bipartite graph $G_{S^{\prime}, R}$ associated with $R$ and $S^{\prime}$. In the second phase (Section 4.2) a solution of $\langle|\Sigma|, m\rangle$-AP is computed starting from a maximum weighted matching of the graph $G_{S^{\prime}, R}$. Section 4.3 is devoted to prove that the solution computed by the algorithm is optimal.

\subsection{Building the graph $G_{R, S^{\prime}}$}

Let us consider a candidate set $S^{\prime}$ of vectors for an optimal solution of $\langle|\Sigma|, m\rangle$-AP. Since $S^{\prime} \subseteq$ $S$, there exist at most $2^{\left|\Sigma^{*}\right|^{m}}$ possible candidate sets of rows $S^{\prime}$, therefore our FPT algorithm computes each candidate set $S^{\prime}$ and verifies if there exists a solution $\Pi_{S^{\prime}}$ with cost at most $e$. In order to verify if such a solution exists, the algorithm builds a bipartite graph $G_{R, S^{\prime}}$, as described in this section. The intuitive idea behind the graph is that edges of the graph correspond to possible ways of assigning each row in $R$ to a resolution vector $x \in S^{\prime}$. Rows assigned to the same resolution vector $x \in S^{\prime}$ are clustered in the solution $\Pi_{S^{\prime}}$.

The construction of the vertex set of the graph is based on a a partition of $R$ into two disjoint sets called $R_{\text {safe }}$ and $R_{\text {dist }}$ (that is $R_{\text {dist }}=R \backslash R_{\text {safe }}$ ). The set $R_{\text {safe }}$ consists of those rows $r \in R$ belonging to the group $g$ such that: $s(g) \geq k$, that is $r$ belongs to a group of at least $k$ identical rows, and there exists a row $r_{j} \in S^{\prime}$, such that $r_{j}$ and $r(g)$ are the same vector. Notice that only rows in $R_{\text {safe }}$ might have no suppressed entry in a solution $\Pi_{S^{\prime}}$.

The vertex set of $G_{R, S^{\prime}}=(V, E)$ has 6 sets. Two sets $\left(R_{d i s t}^{l}, R_{\text {dist }}^{r}\right)$ consist of vertices associated with the rows in $R_{\text {dist }}$, three sets $\left(R_{\text {safe }}^{l l}, R_{\text {safe }}^{l}, R_{\text {safe }}^{r}\right)$ consist of vertices associated with the rows 
in $R_{\text {safe }}$, and a final set called $T$ consists of vertices associated with the rows in $S^{\prime}$. In the latter case notice that for each row $x$ in $S^{\prime}$ there exist $k$ vertices in $T$ to ensure that the cluster associated with $x$ has size at least $k$. The vertex set is defined as follows:

- for each row $x \in R_{d i s t}$, there is a corresponding vertex $R_{d i s t}^{l}(x)$ in $R_{d i s t}^{l}$ and a corresponding vertex $R_{d i s t}^{r}(x)$ in $R_{d i s t}^{r}$;

- for each group $g$ consisting of the set of rows $\left\{x_{1}, x_{2}, \ldots, x_{s(g)}\right\}$, where each $x_{i} \in R_{\text {safe }}$, $1 \leq i \leq s(g)$, there are $k$ corresponding vertices in $R_{\text {safe }}^{\prime l}$, (such vertices are denoted by $\left.R_{\text {safe }}^{\prime l}(g, 1), \ldots, R_{\text {safe }}^{\prime l}(g, k)\right)$, exc $(g)$ corresponding vertices in $R_{\text {safe }}^{l}$ (such vertices are denoted by $R_{\text {safe }}^{l}(g, 1), \ldots, R_{\text {safe }}^{l}(g, \operatorname{exc}(g))$, and $\operatorname{exc}(g)$ corresponding vertices in $R_{\text {safe }}^{r}$ (such vertices are denoted by $R_{\text {safe }}^{r}(g, 1), \ldots, R_{\text {safe }}^{r}(g, \operatorname{exc}(g))$;

- for each row $x \in S^{\prime}$, there are $k$ corresponding vertices in $T$ (such vertices are denoted by $T(x, 1), \ldots, T(x, k))$.

Notice that our graph $G_{R, S^{\prime}}$ is edge-weighted. Let $w_{h}$ be the weight function assigning a positive weight to each edge of $G_{R, S^{\prime}}$. Given the set of edges $E^{\prime} \subseteq E$, we denote by $w_{h}\left(E^{\prime}\right)=$ $\sum_{e \in E^{\prime}} w_{h}(e)$.

First, notice that the set $S^{\prime}$ consists of two disjoint sets: the set $S_{\text {safe }}^{\prime}$ consists of those rows in $S^{\prime}$ that have no suppressions, while $S_{\text {cost }}^{\prime}=S^{\prime} \backslash S_{\text {safe }}^{\prime}$. Each edge connects a vertex of $R_{\text {safe }}^{\prime l} \cup$ $R_{\text {safe }}^{l} \cup R_{\text {dist }}^{l}$ with a vertex of $R_{\text {safe }}^{r} \cup R_{\text {dist }}^{r} \cup T$, hence the graph $G_{R, S^{\prime}}$ is bipartite. The set $S^{\prime}$ consists of two disjoint sets: the set $S_{\text {safe }}^{\prime}$ consists of those rows in $S^{\prime}$ that have no suppressions, while $S_{\text {cost }}^{\prime}=S^{\prime} \backslash S_{\text {safe }}^{\prime}$. Intuitevely, each edge represents a possible assignment of a row in $R$ to a resolution vector in $S^{\prime}$.

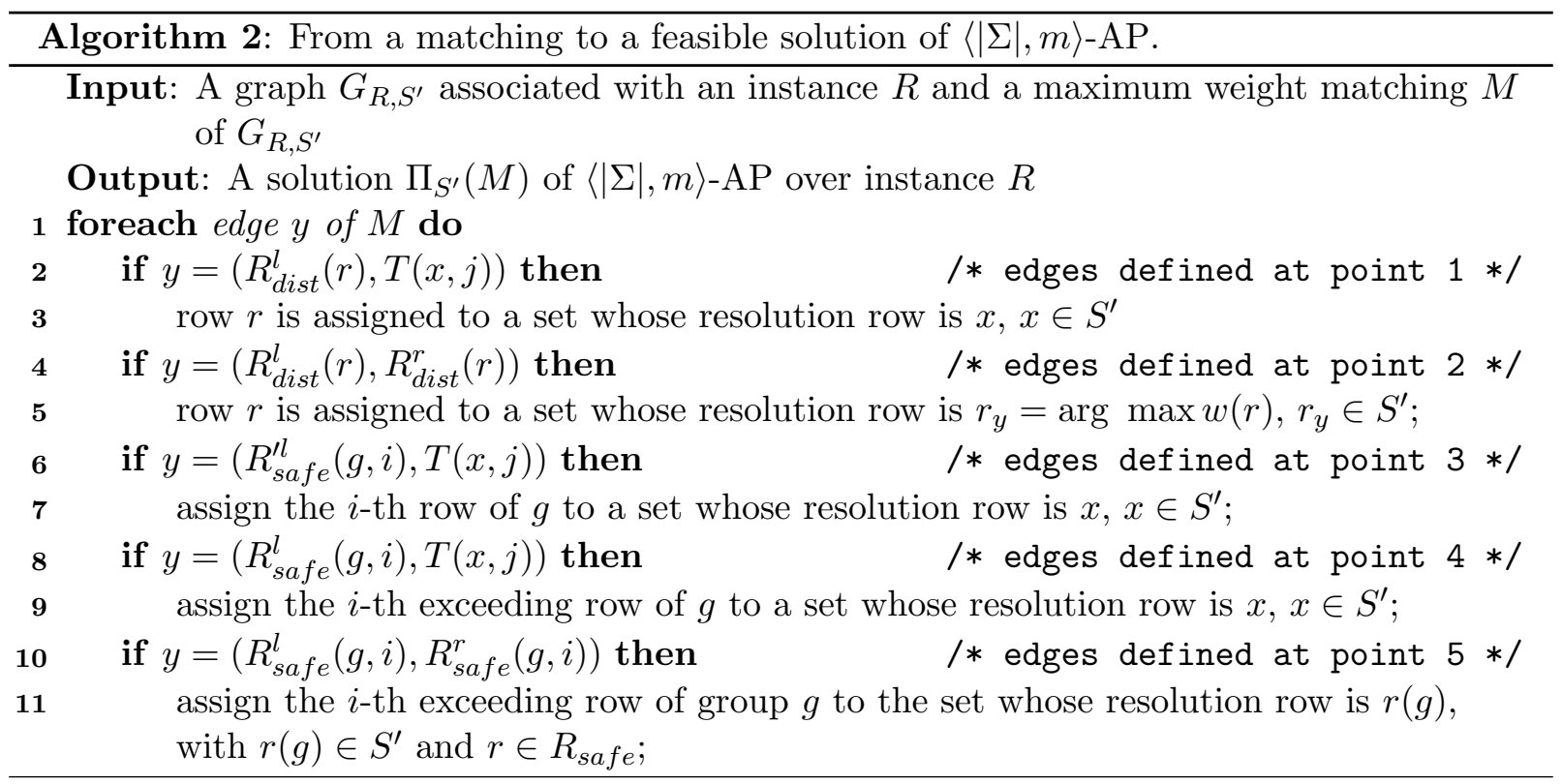

Now we are ready to define formally the set of edges $E$ of $G_{R, S^{\prime}}$ and the weight function $w_{h}$. There are five possible kinds of edges. 
1. Let $r$ be a row of $R_{\text {dist }}$, and let $x$ be a row in $\operatorname{Comp}\left(r, S^{\prime}\right) \cap S_{\text {cost }}^{\prime}$. Then there is an edge $y=\left(R_{\text {dist }}^{l}(r), T(x, j)\right)$, for each $1 \leq j \leq k$, with weight $w_{h}(y)=w^{\prime}(x)$.

2. Let $r$ be a row in $R_{d i s t}$. Then there is an edge $y=\left(R_{d i s t}^{l}(r), R_{d i s t}^{r}(r)\right)$ with weight $w_{h}(y)=$ $w(r)$.

3. Let $g$ be a group consisting of rows $\left\{r_{1}, \ldots, r_{s(g)}\right\}$, where $r_{i}$, for each $i$ with $1 \leq i \leq s(g)$, is a row of $R_{\text {safe }}$; let $r^{\prime}$ be the resolution vector of $S_{\text {safe }}^{\prime}$ identical to $r(g)$. Then there is an edge $y_{i}=\left(R_{\text {safe }}^{\prime l}(g, i), T\left(r^{\prime}, i\right)\right)$, for each $i$ with $1 \leq i \leq k$. All edges $y_{i}$ have weight $w_{h}\left(y_{i}\right)=w^{\prime}\left(r^{\prime}\right)$.

4. Let $g$ be a group consisting of rows $\left\{r_{1}, \ldots, r_{s(g)}\right\}$, where $r_{i}$, for each $i$ with $1 \leq i \leq s(g)$, is a row of $R_{\text {safe }}$; let $x$ be a row in $\operatorname{Comp}\left(r(g), S^{\prime}\right) \cap S_{\text {cost }}^{\prime}$. Then there is an edge $y_{i, j}=$ $\left(R_{\text {safe }}^{l}(g, i), T(x, j)\right)$, for each $i$ with $1 \leq i \leq \operatorname{exc}(g)$ and for each $j$ with $1 \leq j \leq k$. All edges $y_{i, j}$ have weight $w_{h}\left(y_{i, j}\right)=w^{\prime}(x)$.

5. Let $g$ be a group consisting of rows $\left\{r_{1}, \ldots, r_{s(g)}\right\}$, where $r_{i}, 1 \leq i \leq s(g)$, is a row of $R_{s a f e}$. Then there is an edge $y_{i}=\left(R_{\text {safe }}^{l}(g, i), R_{\text {safe }}^{r}(g, i)\right)$ for each $i$ with $1 \leq i \leq \operatorname{exc}(g)$. All edges $y_{i}$ have weight $w_{h}\left(y_{i}\right)=w(r(g))$.

\subsection{Computing a solution of $\langle|\Sigma|, m\rangle$-AP}

In this section we prove in Lemma 6 that $\Pi_{S^{\prime}}(M)$ is a clustering of the rows in $R$ that is a feasible solution for the $\langle|\Sigma|, m\rangle$-AP problem. See Fig. 4.2 for an example.

Since $G_{R, S^{\prime}}$ bipartite, we can efficiently compute a maximum weight matching $M$ of $G_{R, S^{\prime}}$ [17]. Given a matching $M$ of the graph $G_{R, S^{\prime}}$, Algorithm 2 computes in polynomial time a clustering $\Pi_{S^{\prime}}(M)$ of the rows in $R$. Informally, the clustering is computed by assigning the rows in $R$ to the resolution vector in $S^{\prime}$, using the edges in the matching $M$.

Notice that, each vertex $R_{\text {safe }}^{l}(r, i)$ has only the edge $\left(R_{\text {safe }}^{l}(r, i), T(r, i)\right)$ on it, hence we can always add those edges to any matching ${ }^{1}$. Let $M$ be a matching of $G_{R, S^{\prime}}$ and let $v$ be a vertex of $G_{R, S^{\prime}}$, then we say that $v$ is covered by a matching $M$ if there exists an edge of $M$ for which $v$ is one of its endpoints. Moreover, we will say that $M$ is feasible if all vertices in $T$ are covered by $M$. When a matching $M$ covers all vertices in $R_{\text {dist }}^{l} \cup R_{\text {safe }}^{l}$ and is feasible, it is defined as a complete matching. Let $\Pi$ be a clustering of an instance $R$ of the $\langle|\Sigma|, m\rangle$-AP problem. Then $\Pi$ is feasible if and only if each set of the partition $\Pi$ contains at least $k$ rows. The next part of this section is devoted to show that every maximum weight matching $M$ is complete and that clustering $\Pi_{S^{\prime}}(M)$ is feasible. First, we will show in the next two lemmata that, given $W^{\prime}=k \sum_{r_{x} \in T} w^{\prime}\left(r_{x}\right), W^{\prime}$ is a threshold that distinguishes between matchings that are feasible and those that are not.

Lemma 4. Let $M$ be a matching of $G_{R, S^{\prime}}$, let $X$ be the subset of $T$ consisting of the vertices of $T$ that are covered by $M$, and let $M_{1}$ be the subset of the edges of $M$ that have one endpoint in $X$. Then the total weight of the edges in $M_{1}$ is exactly $\sum_{T(t, i) \in X} w^{\prime}(t)$.

Proof. It is an immediate consequence of the observation that all edges where an endpoint is $T(t, j)$ have the same weight $w^{\prime}(t)$, with $t \in S^{\prime}$.

\footnotetext{
${ }^{1}$ Notice that these connected components are introduced only to simplify the relationship between a matching $M$ and the corresponding solution $\Pi_{S^{\prime}}(M)$ of $\langle|\Sigma|, m\rangle$-AP
} 


\begin{tabular}{clll}
\multicolumn{4}{c}{ Rows $R$} \\
\hline \hline Name & Data & $w$ & Group \\
\cline { 1 - 3 }$r_{1}$ & aaa & 3 & \\
\cline { 1 - 3 }$r_{2}$ & aaa & 3 & \\
\cline { 1 - 3 }$r_{3}$ & aaa & 3 & \\
\cline { 1 - 3 }$r_{4}$ & aaa & 3 & \\
\cline { 1 - 3 }$r_{5}$ & aba & 2 & $g_{2}$ \\
\hline$r_{6}$ & bbb & 2 & $g_{3}$ \\
\hline$r_{7}$ & bbc & 2 & $g_{4}$ \\
\hline
\end{tabular}

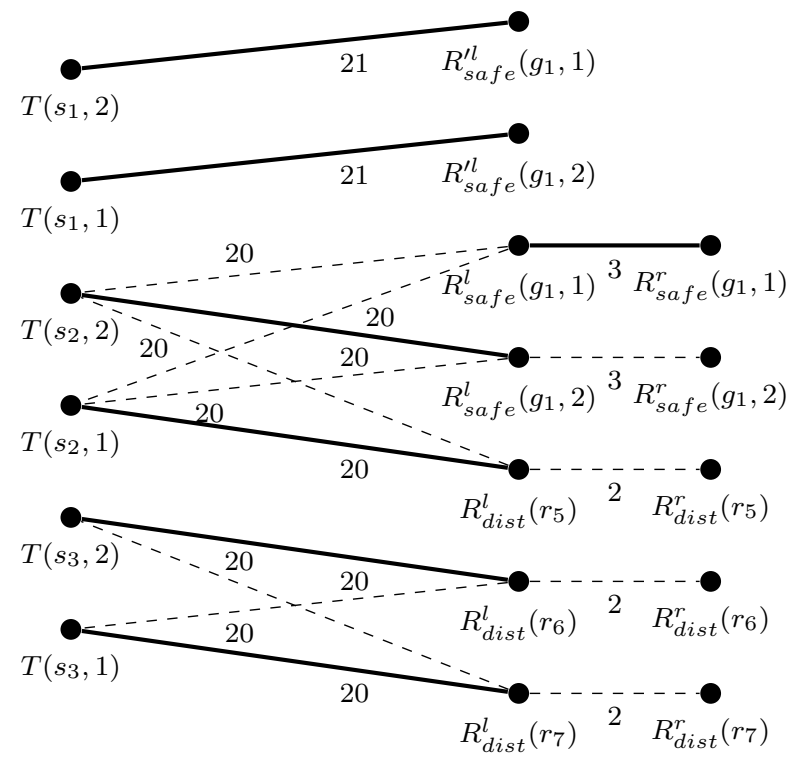

\begin{tabular}{cll}
\multicolumn{3}{c}{ Resolution vectors $S^{\prime}$} \\
\hline \hline Name & Vectors & $w$ \\
\hline$s_{1}$ & aaa & 21 \\
\hline$s_{2}$ & $\mathrm{a}^{*} \mathrm{a}$ & 20 \\
\hline$s_{3}$ & $\mathrm{bb}^{*}$ & 20 \\
\hline
\end{tabular}

Figure 1: An instance $R$ of $\langle|\Sigma|, m\rangle$-AP, with $k=2$ and $m=3$, a resolution vector set $S^{\prime}$ and the associated graph $G_{R, S^{\prime}}$. The thick edges are a maximum weight matching of $G_{R, S^{\prime}}$. The corresponding solution is made of the sets $\left\{r_{1}, r_{2}, r_{3}\right\}$ (cost 0$),\left\{r_{4}, r_{5}\right\}$ (cost 2$),\left\{r_{6}, r_{7}\right\}$ (cost 2).

Lemma 5. Let $M$ be a matching of $G_{R, S^{\prime}}$ and let $M_{1}$ be the subset of the edges of $M$ that have one endpoint in $T$. Then the total weight of the edges in $M_{1}$ is at least $W^{\prime}=k \sum_{r \in S^{\prime}} w^{\prime}(r)$ if and only if $M$ is feasible.

Proof. Let $M_{1}$ be the subset of the edges of $M$ that have one endpoint in $T$, and let $W_{1}$ be the total weight of edges in $M_{1}$. An immediate consequence of Lemma 4 is that $W_{1}=W^{\prime}$ if and only if $M_{1}$ is feasible. Assume now that $M$ is not feasible, then there exists at least one vertex $S^{\prime}(x, j) \in T$ that is not covered by $M$. Again, a consequence of Lemma 4 is that $W_{1} \leq W^{\prime}-w^{\prime}(x)$. Let $M_{2}$ be the set $M \backslash M_{1}$. By construction, $w^{\prime}(x)>W$ and $W$ is an upper bound on the total weight of $M_{2}$, therefore $W_{1}+w_{h}\left(M_{2}\right)<W^{\prime}$, completing the proof.

Using Lemmata 4 and 5 , we can prove Lemma 6 .

Lemma 6. Let $M$ be a maximum weight matching of $G_{R, S^{\prime}}$, then $M$ is complete and the solution $\Pi_{S^{\prime}}(M)$ computed by Algorithm 2 is feasible.

\subsection{Proving the optimality of $\Pi_{S^{\prime}}(M)$}

This section is devoted to prove that, starting from a maximum weight matching $M$, Algorithm 2 computes an optimal solution $\Pi_{S^{\prime}}(M)$ of $\langle|\Sigma|, m\rangle$-AP. In order to prove that any maximum weight matching $M$ of the graph $G_{R, S^{\prime}}$ leads to an optimal solution of $\langle|\Sigma|, m\rangle$-AP over instance $R$, we are going to prove that $\sum_{(u, v) \in M} w_{h}((u, v)) \geq(W+1) k\left|S^{\prime}\right|+m\left|R_{\text {dist }}^{l} \cup R_{\text {safe }}^{l} \cup R_{\text {safe }}^{\prime l}\right|-e$ if and only if $\langle|\Sigma|, m\rangle$-AP over instance $R$ admits a solution with cost not greater than $e$, and such solution is computed by applying Algorithm 2. Such result will be obtained through a sequence of technical lemmata. 
Since $M$ is a maximum weighted matching, we can assume by Lemma 6 that $M$ is complete. Given a complete matching $M$, we denote by $M(T)$ the set of edges of $M$ with one endpoint in $R_{\text {dist }}^{l} \cup R_{\text {safe }}^{l} \cup R_{\text {safe }}^{l l}$ and one endpoint in $T$, while we denote by $M(L)$ the set of those edges of $M$ that have one endpoint in $R_{\text {dist }}^{l} \cup R_{\text {safe }}^{l}$ and one endpoint in $R_{\text {dist }}^{r} \cup R_{\text {safe }}^{r}$. Furthermore, let us denote by $V(T)$ the set of vertices of $R_{\text {dist }}^{l} \cup R_{\text {safe }}^{l} \cup R_{\text {safe }}^{\prime l}$ that are endpoints of an edge in $M(T)$ and by $V(L)$ the set of vertices of $R_{\text {dist }}^{l} \cup R_{\text {safe }}^{l}$ that are endpoints of an edge in $M(L)$. Notice that by definition of $V(L)$ and, by definition of complete matching, $V(T) \cup V(L)=R_{\text {dist }}^{l} \cup R_{\text {safe }}^{l} \cup R_{\text {safe }}^{\prime l}$. Finally, let us denote by $R(L)$ the set of rows in $R$ associated with the vertices in $V(L)$. Lemma 7 shows how the weight of a complete matching $M$ is related to the edge weights of $G_{R, S^{\prime}}$.

Lemma 7. Let $M$ be a complete matching of $G_{R, S^{\prime}}$, and let $w_{h}(M)$ be the total weight of $M$. Then $w_{h}(M)=k \sum_{r \in S^{\prime}}(W+m-\operatorname{del}(r)+1)+\sum_{r \in R(L)}(m-\operatorname{del}(r))=(W+1) k\left|S^{\prime}\right|+m \mid R_{d i s t}^{l} \cup$ $R_{\text {safe }}^{l} \cup R_{\text {safe }}^{\prime l} \mid-\left(k \sum_{r \in S^{\prime}} \operatorname{del}(r)+\sum_{r \in R(L)} \operatorname{del}(r)\right)$.

In the next two lemmata, we will show that: (i) given an instance $R$ of $\langle|\Sigma|, m\rangle$-AP, if there exists a solution of $\langle|\Sigma|, m\rangle$-AP over $R$ that suppresses at most $e$ entries then the graph $G_{R, S^{\prime}}$ associated with $R$ admits a complete matching of $G_{R, S^{\prime}}$ with total weight $w_{G}(M) \geq(W+1) k\left|S^{\prime}\right|+$ $m\left|R_{\text {dist }}^{l} \cup R_{\text {safe }}^{l} \cup R_{\text {safe }}^{\prime l}\right|-e$; (ii) given a complete matching of the graph $G_{R, S^{\prime}}$ of total weight $w_{G}(M) \geq(W+1) k\left|S^{\prime}\right|+m\left|R_{\text {dist }}^{l} \cup R_{\text {safe }}^{l} \cup R_{\text {safe }}^{\prime l}\right|-e$, Algorithm 2 returns a solution $\Pi_{S^{\prime}}(M)$ of $\langle|\Sigma|, m\rangle$-AP that suppresses at most $e$ entries. These lemmata, coupled with Lemma 66, prove the correctness of Algorithm 2 in Theorem 10.

Lemma 8. Let $R$ be an instance of $\langle|\Sigma|, m\rangle-A P$, let $\Pi_{S^{\prime}}$ be a feasible solution of $\langle|\Sigma|, m\rangle-A P$ over instance $R$ that suppresses at most e entries, let $G_{R, S^{\prime}}$ be the graph associated with $R$ and $S^{\prime}$. Then there exists a complete matching of $G_{R, S^{\prime}}$ with total weight $w_{G}(M) \geq(W+1) k\left|S^{\prime}\right|+$ $m\left|R_{\text {dist }}^{l} \cup R_{\text {safe }}^{l} \cup R_{\text {safe }}^{\prime l}\right|-e$.

Lemma 9. Let $R$ be an instance of $\langle|\Sigma|, m\rangle-A P$, let $G_{R, S^{\prime}}$ be the graph associated with $R$, and let $M$ be a complete matching of $G_{R, S^{\prime}}$ of weight $w_{h}(M) \geq(W+1) k\left|S^{\prime}\right|+m\left|R_{\text {dist }}^{l} \cup R_{\text {safe }}^{l} \cup R_{\text {safe }}^{\prime l}\right|-e$. Then, starting from the matching $M$ of $G_{R, S^{\prime}}$, Algorithm 2 computes a feasible solution $\Pi_{S^{\prime}}(M)$ of $\langle|\Sigma|, m\rangle$-AP over instance $R$, where there are at most e suppressions.

Proof. Since $M$ is complete, for each vertex $T(x, j)$ of $T$, with $1 \leq j \leq k$, there exists an edge $(v, T(x, j)) \in M$ for some $v \in\left(R_{\text {dist }}^{l} \cup R_{\text {safe }}^{l} \cup R_{\text {safe }}^{\prime l}\right)$. Then Algorithm 2 defines a solution $\Pi_{S^{\prime}}(M)$ for $\langle|\Sigma|, m\rangle$-AP assigning, for each edge $(v, T(x, j))$, the row $r$ corresponding to vertex $v$ to the set that has resolution vector $x$. More precisely, row $r$ is defined by Algorithm 2 as the $j$-th element of the set that has resolution vector $x$. Therefore each set associated with a resolution row $x \in S^{\prime}$ will consist of at least $k$ rows compatible with $x$. Hence $\Pi_{S^{\prime}}(M)$ is a feasible solution.

Recall that $M$ has a total weight of at least $(W+1) k\left|S^{\prime}\right|+m\left|R_{\text {dist }}^{l} \cup R_{\text {safe }}^{l} \cup R_{\text {safe }}^{\prime l}\right|-e$. We will prove that $\Pi_{S^{\prime}}(M)$ induces at most $e$ suppressions. By Lemma 7, $w_{h}(M)=k \sum_{r \in S^{\prime}}(W+$ $m-\operatorname{del}(r)+1)+\sum_{r \in R(L)} m-\operatorname{del}(r)=(W+1) k\left|S^{\prime}\right|+m\left|R_{\text {dist }}^{l} \cup R_{\text {safe }}^{l} \cup R_{\text {safe }}^{l}\right|-\left(k \sum_{r \in S^{\prime}} \operatorname{del}(r)+\right.$ $\left.\sum_{r \in R(L)} \operatorname{del}(r)\right) \geq(W+1) k\left|S^{\prime}\right|+m\left|R_{\text {dist }}^{l} \cup R_{\text {safe }}^{l} \cup R_{\text {safe }}^{l}\right|-e$ where $k \sum_{r \in S^{\prime}} \operatorname{del}(r)+\sum_{r \in R(L)} \operatorname{del}(r) \leq$ $e$. Notice that, by definition of $\Pi_{S^{\prime}}(M)$, each vertex of $V(T)$ corresponds to a row in $R$ assigned to a set with a resolution vector in $S^{\prime}$. Such rows associated with $V(T)$ induce a cost in $\Pi_{S^{\prime}}(M)$ of $k \sum_{r \in S^{\prime}} \operatorname{del}(r)$. Furthermore, the vertices of $V(L)$ corresponds to rows of $R$ inducing a cost of at most $\sum_{r \in R(L)} \operatorname{del}(r)$. Therefore $\Pi_{S^{\prime}}(M)$ induces $k \sum_{r \in S^{\prime}} \operatorname{del}(r)+\sum_{r \in R(L)} \operatorname{del}(r) \leq e$ suppressions. 
Theorem 10. Let $R$ be an instance of $\langle|\Sigma|, m\rangle-A P$. Then Algorithm 1 returns a solution $\Pi_{S^{\prime}}(M)$ of cost at most $e$ if and only if such a solution exists.

Proof. By Lemma 6, $\Pi_{S^{\prime}}(M)$ is feasible. Hence if $\Pi_{S^{\prime}}(M)$ suppresses at most $e$ entries, then $\langle|\Sigma|, m\rangle$-AP admits a solution of cost at most $e$. On the other hand, by Lemma 8 , if there exists a solution $\Pi^{\prime}$ of $R$ that suppresses at most $e$ entries, then there exists a feasible matching $M$ with weight $w_{G}(M) \geq(W+1) k\left|S^{\prime}\right|+m\left|R_{\text {dist }}^{l} \cup R_{\text {safe }}^{l} \cup R_{\text {safe }}^{l l}\right|-e$. Then, by Lemma 9, Algorithm 1 returns a solution $\Pi_{S^{\prime}}(M)$ of $\langle|\Sigma|, m\rangle$-AP that suppresses at most $e$ entries.

If $\langle|\Sigma|, m\rangle$-AP admits a solution that suppresses at most $e$ entries, then there exists a set $S^{*}$ of resolution vectors such that $\Pi_{S^{*}}$ is a solution for $\langle|\Sigma|, m\rangle$-AP with resolution vectors $S^{*}$ with the property that $\Pi_{S^{*}}$ suppresses at most $e$ entries. Now, there exist $O\left(2^{(|\Sigma|+1)^{m}}\right)$ possible sets of resolution vectors and the construction of graph $G_{R, S^{\prime}}$ requires $O\left(k\left|S^{*}\right||R|\right) \leq O(k e|R|) \leq$ $O\left(k m n^{2}\right)$. A maximum matching $M$ of a bipartite graph can be computed in polynomial time [17] and starting from $M$, we can compute a solution of the $\langle|\Sigma|, m\rangle$-AP in time $O(|M|) \leq O(m)$. Hence the overall time complexity of the algorithm is $O\left(2^{(|\Sigma|+1)^{m}} k m n^{2}\right)$.

\section{APX-hardness of 3-AP(3)}

In this section we investigate the computational and approximation complexity of 3-AP(3), that is $k$-AP when each row consists of exactly 3 columns and $k=3$. We show that $3-\mathrm{AP}(3)$ is $\mathrm{APX}$ hard via an L-reduction from Minimum Vertex Cover on Cubic Graphs (MVCC), which is known to be APX-hard [4]. Due to page limit, we only sketch the proof. The MVCC problem, given a cubic graph $G=(V, E)$, asks for a smallest $C \subseteq V$ such that each edge of $G$ has at least one of its endpoints in $C$.

Let $G=(V, E)$ be instance of MVCC, where $|V|=n$ and $|E|=m$. The reduction builds an instance $R$ of $3-\mathrm{AP}(3)$ associating with each vertex $v_{i} \in V$ a set $R_{i}$ consisting of 9 rows, and with each edge $e=\left(v_{i}, v_{j}\right) \in E$ a set $E_{i, j}$ consisting of 7 rows. Finally, a set $X$ of 3 more rows is added to $R$.

Now we can describe formally our reduction. Let $R_{i}$ be the set of rows associated with vertex $v_{i} \in V$. The rows in $R_{i}$ have values over an alphabet $\Sigma_{i}=\left\{\sigma_{i}, \sigma_{i, 1}, \sigma_{i, 2}, \sigma_{i, 3}\right\}$. The set $R_{i}$ consists of 9 rows belonging to 6 groups, denoted by $g_{1}\left(v_{i}\right), \ldots, g_{6}\left(v_{i}\right)$, of identical rows. The representative rows of groups $g_{1}\left(v_{i}\right), \ldots, g_{6}\left(v_{i}\right)$, and the cardinality of the groups, are defined as follows:

- $r\left(g_{h}\left(v_{i}\right)\right)=\sigma_{i, h} \sigma_{i} \sigma_{i, h}$, with $h \in\{1,2,3\}$; each group $g_{h}\left(v_{i}\right)$, with $h \in\{1,2,3\}$, consists of exactly two rows;

- $r\left(g_{3+h}\left(v_{i}\right)\right)=\sigma_{i} \sigma_{i} \sigma_{i, h}$, with $h \in\{1,2,3\}$; each group $g_{3+h}\left(v_{i}\right)$, with $h \in\{1,2,3\}$, consists of exactly one row.

Notice that given two rows $r, r^{\prime}$ belonging to different groups of $R_{i}, H\left(r, r^{\prime}\right)=1$ iff $r \in g_{h}\left(v_{i}\right)$, $r^{\prime} \in g_{3+h}\left(v_{i}\right)$ (or the converse) or $r, r^{\prime} \in\left\{g_{4}\left(v_{i}\right), g_{5}\left(v_{i}\right), g_{6}\left(v_{i}\right)\right\}$. Given a group $g_{h}\left(v_{i}\right)$, with $h \in\{1,2,3\}$, each symbol $\sigma_{i, h}$ is called the private symbol of $g_{h}\left(v_{i}\right)$. The groups of rows $g_{j}\left(v_{i}\right)$, with $j \in\{1,2,3\}$, are denoted as the docking groups of $R_{i}$, and each of them is associated with a set $E_{i, h}$ of rows encoding an edge $\left(v_{i}, v_{h}\right)$ of $G$. More precisely, given the set of rows $E_{i, j}$, we denote by $d_{i, j}\left(g\left(v_{i}\right)\right)$ the docking group of $R_{i}$ associated with set $E_{i, j}$.

Now, let us build the set $E_{i, j}$ of rows associated with an edge $\left(v_{i}, v_{j}\right)$. Let $d_{i, j}\left(g\left(v_{i}\right)\right)$ and $d_{i, j}\left(g\left(v_{j}\right)\right)$ be the two docking groups of $R_{i}$ and $R_{j}$ respectively, associated with the set $E_{i, j}$. 
Let $\sigma_{i, x}$ and $\sigma_{j, y}$ be the private symbols of groups $d_{i, j}\left(g\left(v_{i}\right)\right)$ and $d_{i, j}\left(g\left(v_{j}\right)\right)$ respectively. The set $E_{i, j}$ consists of 7 rows distributed in 6 groups. The rows of $E_{i, j}$ have values over alphabet $\Sigma_{i, j}=\left\{\sigma_{i, x}, \sigma_{j, y}, \sigma_{i, j}, \sigma_{i, j, 4}, \sigma_{i, j, 5}, \sigma_{i, j, 6}\right\}$. Let us define the representative rows and the cardinality of the groups in $E_{i, j}$ :

- $r\left(g_{1}\left(v_{i}, v_{j}\right)\right)=\sigma_{i, x} \sigma_{i, j} \sigma_{i, x}$; group $g_{1}\left(v_{i}, v_{j}\right)$ consists of a single row;

- $r\left(g_{2}\left(v_{i}, v_{j}\right)\right)=\sigma_{i, x} \sigma_{i, j} \sigma_{j, y}$; group $g_{2}\left(v_{i}, v_{j}\right)$ consists of two rows;

- $r\left(g_{3}\left(v_{i}, v_{j}\right)\right)=\sigma_{j, y} \sigma_{i, j} \sigma_{j, y}$; group $g_{3}\left(v_{i}, v_{j}\right)$ consists of a single row;

- $r\left(g_{t}\left(v_{i}, v_{j}\right)\right)=\sigma_{i, j, t} \sigma_{i, j} \sigma_{i, j, t}$, with $t \in\{4,5,6\}$; each group $g_{t}\left(v_{i}, v_{j}\right)$, with $t \in\{4,5,6\}$, consists of a single row.

The group of $E_{i, j}$ that has two occurrences of symbol $\sigma_{i, x}$ shared with $d_{i, j}\left(g\left(v_{i}\right)\right)$ is called the $i$-group of set $E_{i, j}$, and is denoted as $g^{i}\left(v_{i}, v_{j}\right)$. Notice that, given two rows $r, r^{\prime}$ of $R_{i}, E_{i, j}$ respectively, then $H\left(r, r^{\prime}\right)=1$ iff $r \in d_{i, j}\left(g\left(v_{i}\right)\right)$ and $r^{\prime} \in g^{i}\left(v_{i}, v_{j}\right)$.

Finally, a set $X$ of 3 rows $x_{1}, x_{2}, x_{3}$ are added to $R$. The rows in $X$ have values over an alphabet $\Sigma_{x}$ disjoint from any other set $\Sigma_{i}, \Sigma_{i, j}$. Each row $x_{i}=w_{i}^{3}$, and it has Hamming distance 3 from any other row of $R$. Therefore for any set $C$ containing some rows $x_{i}$, all positions of a row in $C$ will be suppressed.

Now, consider the set $R_{i}$. The following lemma gives a lower bound on the cost of an optimal solution of $3-\mathrm{AP}(3)$ over instance $R_{i}$.

Lemma 11. Let $R_{i}$ be a set of rows, then an optimal solution of $3-A P(3)$ over instance $R_{i}$ has a cost of at least 9 .

The main idea of the reduction is showing that we can consider a set of solutions, called canonical solutions, that is solutions where:

(i) $\Pi$ contains exactly one cluster $X$ containing only suppressed entries;

(ii) each set $R_{i}$ is associated with either a type $a$ or a type $b$ solution (to be defined later), eventually with the contribution of some rows in the sets $E_{i, j}$ for a type $b$ solution;

(iii) two sets $R_{i}, R_{j}$ are associated with a type $b$ solution only if there is no edge set $E_{i, j}$ in the instance $R$, that is the corresponding vertices $v_{i}, v_{j}$ are not adjacent in $G$;

(iv) either an edge set is part of a type $b$ solution of some set $R_{i}$ and has a total cost of 10 or it has a total cost of 11.

Notice that, by construction, in a canonical solution, rows $x_{1}, x_{2}, x_{3} \in X$.

Let us define the notions of type $a$ and type $b$ solution. Given a set $R_{i}$ and the edge sets $E_{i, j}, E_{i, h}, E_{i, l}$, a type $a$ solution for $R_{i}$ consists of three sets $S_{i, 1}, S_{i, 2}, S_{i, 3}$, where $S_{i, t}=g_{t}\left(v_{i}\right) \cup$ $g_{t+3}\left(v_{i}\right)$, while a type $b$ solution consists of the following sets: (i) three sets $d_{i, j}\left(g\left(v_{i}\right)\right) \cup g^{i}\left(v_{i}, v_{j}\right)$, $d_{i, h}\left(g\left(v_{i}\right)\right) \cup g^{i}\left(v_{i}, v_{h}\right), d_{i, l}\left(g\left(v_{i}\right)\right) \cup g^{i}\left(v_{i}, v_{l}\right)$; (ii) $g_{4}\left(v_{i}\right) \cup g_{5}\left(v_{i}\right) \cup g_{6}\left(v_{i}\right)$.

Lemma 12 is the main technical contribution of this section.

Lemma 12. Let $\Pi$ be a solution of $3-A P(3)$ over instance $R$. Then we can compute in polynomial time a canonical solution $\Pi^{\prime}$ of $3-A P(3)$ over instance $R$ such that $c\left(\Pi^{\prime}\right) \leq c(\Pi)$.

Sketch of the proof. By direct inspection, it is immediate to notice that type $a$ and type $b$ solutions induce 9 suppression in rows of $R_{i}$ hence, by Lemma 11, they are optimal for $R_{i}$. The next step is computing in polynomial time a solution $\Pi^{\prime \prime}$ such that each set $R_{i}$ is associated in $\Pi^{\prime \prime}$ only with either a type $a$ or type $b$ solution, and such that $c\left(\Pi^{\prime \prime}\right) \leq c(\Pi)$. Such step is obtained by exploiting the optimality of type $a$ and type $b$ solutions for $R_{i}$, and some properties of the instance $R$. 
Then, starting from such solution $\Pi^{\prime \prime}$, we can compute in polynomial time a canonical solution $\Pi^{\prime}$ such that $c\left(\Pi^{\prime}\right) \leq c\left(\Pi^{\prime \prime}\right)$. The main idea to prove this result is that for any two sets $R_{i}, R_{j}$, such that both $R_{i}$ and $R_{j}$ are associated with a type $b$ solution in $\Pi^{\prime \prime}$ and $E_{i, j}$ is part of the instance $R$, then we can improve the solution by imposing a type a solution for $R_{i}$.

A consequence of Lemmata 11 and 12 and some properties of the instance $R$, is Lemma 13 .

Lemma 13. Let $\Pi$ be a solution of $3-A P(3)$ over instance $R$ of cost $6|V|+3|C|+11|E|+9$, then we can compute in polynomial time a solution of $M V C C$ over instance $G$ of size $C$.

Proof. Let us consider a canonical solution of 3-AP(3). First ,notice that the three rows $w_{1}, w_{2}$, $w_{3}$ provide together a cost of 9 . Since two sets of rows are associated with a type $b$ solution only if there does not exist a set $E_{i, j}$, on the contrary, given an edge set $E_{i, j}$ at least one of the set $R_{i}$ and $R_{j}$ is associated with a type a solution. Consequently, the set of rows associated with a type $a$ solution corresponds to a vertex cover of the graph $G$.

Now consider the cost of a canonical solution. For each set $R_{i}$ of rows associated with a type $b$ solution, we can show that each of the three edge sets $E_{i, j}, E_{i, h}, E_{i, l}$ has a cost of 10 . Notice that, given an edge set $E_{i, j}$, if both sets $R_{i}, R_{j}$ are associated with type a solutions, then we can show that the edge set $E_{i, j}$ has a cost of 11 . Accounting this decreasing of the cost of the edge sets to the set $R_{i}$ of rows with a type $b$ solution, is equivalent to assign to a type $b$ solution a cost equal to 6 , while a type a solution has a cost equal to 9 .

Similarly to Lemma 13, we can prove that starting from a solution $C$ of MVCC over instance $G$, we can compute in polynomial time a solution $\Pi$ of 3-AP(3) over instance $R$ of cost $6|V|+$ $3|C|+11|E|+9$. Therefore 3-AP(3) is APX-hard.

\section{References}

[1] G. Aggarwal, T. Feder, K. Kenthapadi, S. Khuller, R. Panigrahy, D. Thomas, and A. Zhu. Achieving anonymity via clustering. In PODS, pages 153-162. 2006.

[2] G. Aggarwal, T. Feder, K. Kenthapadi, R. Motwani, R. Panigrahy, D. Thomas, and A. Zhu. Anonymizing tables. In ICDT, LNCS 3363, pages 246-258. 2005.

[3] G. Aggarwal, K. Kenthapadi, R. Motwani, R. Panigrahy, D. Thomas, and A. Zhu. Approximation algorithms for k-anonymity. J. Privacy Technology, 2005.

[4] P. Alimonti and V. Kann. Some APX-completeness results for cubic graphs. Theoretical Computer Science, 237(1-2):123-134, 2000.

[5] G. Ausiello, P. Crescenzi, V. Gambosi, G. Kann, A. Marchetti-Spaccamela, and M. Protasi. Complexity and Approximation: Combinatorial optimization problems and their approximability properties. Springer-Verlag, 1999.

[6] P. Bonizzoni, G. Della Vedova, and R. Dondi. The k-anonymity problem is hard. In FCT, LNCS 5699, pages 26-37. 2009.

[7] R. Chaytor, P. A. Evans, and T. Wareham. Fixed-parameter tractability of anonymizing data by suppressing entries. J. Comb. Optim., 18(4): 362-375, 2009.

[8] R. Downey and M. Fellows. Parameterized Complexity. Springer Verlag, 1999.

[9] R. G. Downey and M. R. Fellows. Fixed-parameter tractability and completeness ii: On completeness for W[1]. Theoretical Computer Science, 141(1\&2):109-131, 1995. 
[10] W. Du, D. Eppstein, M. T. Goodrich, and G. S. Lueker. On the approximability of geometric and geographic generalization and the min-max bin covering problem. In WADS, LNCS 5664, pages 242-253. 2009.

[11] A. Gionis and T. Tassa. k-anonymization with minimal loss of information. TKDD, 21(2): 206-219, 2009.

[12] A. Meyerson, R. Williams. On the complexity of optimal K-anonymity. In PODS, pages 223-228. 2004.

[13] R. Niedermeier. Invitation to Fixed-Parameter Algorithms. Oxford University Press, 2006.

[14] H. Park and K. Shim. Approximate algorithms for k-anonymity. In SIGMOD, pages 67-78. 2007.

[15] P. Samarati. Protecting respondents' identities in microdata release. TKDD, 13:1010-1027, 2001.

[16] P. Samarati and L. Sweeney. Generalizing data to provide anonymity when disclosing information (abstract). In PODS, page 188. 1998.

[17] J. Schwartz, A. Steger and A. Weissl, Fast algorithms for weighted bipartite matching. In WEA, pages 476-487, 2005.

[18] L. Sweeney. k-anonymity: a model for protecting privacy. International Journal on Uncertainty, Fuzziness and Knowledge-based Systems, 10(5):557-570, 2002. 


\section{Appendix}

\section{Proofs of Section 3}

\section{Proof of Lemma 1}

Lemma 14. Let $R$ be the instance of $\langle e\rangle-A P$ associated with $G$ and consider two rows $r, r_{x}(i, j)$ of $R$, such that $r \in R_{0}$ and $r_{x}(i, j) \in R(i, j)$. Then, $r[t] \neq r_{x}(i, j)[t]$, for each $1 \leq t \leq 2 h$.

Proof. By construction, $r[t]=0$ for all $t$ with $1 \leq t \leq 2 h$, while $r_{x}(i, j)[t]=\sigma_{i, j}$.

\section{Proof of Lemma 2}

Lemma 15. Let $G=(V, E)$ be a graph, let $V^{\prime}$ be a h-clique of $G$ and let $R$ be the instance of $\langle e\rangle$-AP associated with $G$. Then we can compute in polynomial time a solution $\Pi$ of $\langle e\rangle$-AP over instance $R$ with cost at most $6 h^{3}$.

Proof. Initially let $\Pi^{\prime}$ be a solution consisting of clusters $R_{0}, R(i, j)$, for each $R(i, j) \in R$. For each $R(i, j)$, let $r_{1}(i, j)$ be the first row of $R(i, j)$. Compute a new solution $\Pi$ consisting of clusters $R_{0}^{\prime}, R^{\prime}(i, j)$, for each $R(i, j) \in R$, where:

- $R^{\prime}(i, j)=R(i, j) \backslash\left\{r_{1}(i, j)\right\}$, for each $v_{i}, v_{j} \in V^{\prime}$

- $R^{\prime}(i, j)=R(i, j)$, for $v_{i} \notin V^{\prime}$ or $v_{j} \notin V^{\prime}$;

- $R_{0}^{\prime}=R_{0} \bigcup_{R(i, j) \in R}\left(R^{\prime}(i, j) \backslash R(i, j)\right)$

Notice that, since $V^{\prime}$ is a $h$-clique, $\left|R_{0}^{\prime}\right|=k$. Moreover, by construction, $|R(i, j)| \geq\left|R^{\prime}(i, j)\right| \geq|R(i, j)|-1$, therefore $\Pi$ is a feasible solution for $R$. Notice also that no entries is suppressed in the rows of each set $R^{\prime}(i, j)$, therefore to determine the cost of $\Pi^{\prime}$ it suffices to determine the number of entries deleted in $R_{0}^{\prime}$, and we will show that such number is exactly $6 h^{3}$.

Indeed, by construction, for each column $t$ of the first $2 h$ columns, and for each row $r \in R_{0}$ and $r_{x}(i, j) \in R(i, j), r[t] \neq r_{x}(i, j)[t]$, hence all the entries of the first $2 h$ columns of the rows in $R_{0}^{\prime}$ must be deleted, resulting in $2 h k$ suppressions. Now let us consider the columns with index $2 h+1 \leq t \leq 2 h+n$ and $v_{t} \in V^{\prime}$. In such positions, all rows of $R_{0}$ are equal to 0 , while all rows in the sets $R(y, t), R(t, y)$ are equal to 1 . Consider the $\frac{h(h-1)}{2}$ of $R_{0}^{\prime} \backslash R_{0}$. As the corresponding edges are incident on a set of $h$ vertex, by construction there exists a set $H$ of exactly $h$ columns, with $H=\{t: 2 h+1 \leq t \leq 2 h+n\}$, where at least one of the rows in $R_{0}^{\prime} \backslash R_{0}$ is equal to 1 , while the rows in $R_{0}$ are all equal to 0 . Since in any other column all rows in $R_{0}^{\prime}$ have value equal to 0 , hence there are additional $h k$ suppressions for the columns with index $2 h+1 \leq t \leq 2 h+n$. Overall, the number of suppressions is $3 h k$ which, by the choice of $k$ is equal to $6 h^{3}$.

\section{Proofs of Section 4}

\section{Proof of Lemma 6}

Lemma 16. Let $M$ be a maximum weight matching of $G_{R, S^{\prime}}$, then the solution $\Pi_{S^{\prime}}(M)$ computed by Algorithm 2 is feasible.

Lemma 6 is a consequence of Lemmata 19 and 17 .

Lemma 17. Let $M$ be a maximum weight matching of $G_{R, S^{\prime}}$, then $M$ is a feasible matching.

Proof. First notice that, as $M$ is feasible, each vertex of $T$ is covered and each vertex $R_{\text {safe }}^{l}(r, j)$, with $1 \leq j \leq k$, is covered by $M$. Assume that $M$ is not complete and that a vertex $R_{\text {dist }}^{l}(r)$ of $R_{\text {dist }}^{l}$ (resp. $R_{\text {safe }}^{l}(r, k+j)$, with $1 \leq j \leq \operatorname{exc}(g)$, of $\left.R_{\text {safe }}^{l}\right)$ is not matched. Then, by construction, also the vertex 
$R_{\text {dist }}^{r}(r)$ of $R_{\text {dist }}^{r}$ (resp. $R_{\text {safe }}^{r}(r, j)$ of $R_{\text {safe }}^{r}$ ) is not covered by $M$, as $R_{\text {dist }}^{l}(r)\left(\right.$ resp. $\left.R_{\text {safe }}^{l}(r, k+j)\right)$ is the only vertex adjacent to $R_{\text {dist }}^{r}(r)$ (resp. $\left.R_{\text {safe }}^{r}(r, j)\right)$ in $G_{R, S^{\prime}}$. Hence we can compute the matching $M^{\prime}$ by adding all the edges of $M$ to $M^{\prime}$ and by adding edges $\left(R_{\text {dist }}^{l}(r), R_{\text {dist }}^{r}(r)\right),\left(\operatorname{resp} .\left(R_{\text {safe }}^{l}(r, k+j), R_{\text {safe }}^{r}(r, j)\right)\right)$ for each vertex $R_{\text {dist }}^{l}(r)$ (resp. $\left.R_{\text {safe }}^{l}(r, k+j)\right)$ not covered by $M$.

Lemma 18. Let $M$ be a feasible matching of $G_{R, S^{\prime}}$, if $M$ is not complete, then we can compute in polynomial time a complete matching $M^{\prime}$, such that $w_{h}\left(M^{\prime}\right)>w_{h}(M)$.

As a consequence of Lemma 18, we assume in what follows that any matching $M$ is complete. Furthermore, we can prove the following result.

Lemma 19. Let $M$ be a complete matching of $G_{R, S^{\prime}}$. Then Algorithm 2 computes in polynomial time a feasible clustering $\Pi_{S^{\prime}}(M)$.

Proof. Since $\Pi_{S^{\prime}}(M)$ feasible, all vertices in $T$ are covered by $M$. Furthermore, we can assume, by Lemma 18. that each vertex in $R_{\text {dist }}^{l} \cup R_{\text {safe }}^{l}$ is covered by $M$. Hence each row in $R$ is assigned by Algorithm 2 to a set whose resolution vector is $S^{\prime}$. Furthermore Algorithm 2 assigns to each set with resolution vector $x \in S^{\prime}$ at least $k$ rows. Hence the clustering $\Pi_{S^{\prime}}(M)$ computed by Algorithm 2 is feasible.

\section{Proof of Lemma 7}

Lemma 20. Let $M$ be a complete matching of $G_{R, S^{\prime}}$, then the total weight of $M, w_{h}(M)$, is equal to $k \sum_{r \in S^{\prime}}(W+m-\operatorname{del}(r)+1)+\sum_{r \in R(L)}(m-\operatorname{del}(r))=(W+1) k\left|S^{\prime}\right|+m\left|R_{\text {dist }}^{l} \cup R_{\text {safe }}^{l}\right|-\left(k \sum_{r \in S^{\prime}} \operatorname{del}(r)+\right.$ $\left.\sum_{r \in R(L)} \operatorname{del}(r)\right)$.

Proof. The total weight $w_{h}(M)$ of the matching $M$ is defined as

$$
w_{h}(M)=\sum_{(u, v) \in M(T)} w_{h}((u, v))+\sum_{(u, v) \in M(L)} w_{h}(u, v) .
$$

By Lemma 5 and by definition of the weight function $w_{h}$, it follows that

$$
w_{h}(M)=k \sum_{r \in S^{\prime}} w^{\prime}(r)+\sum_{r \in R(L)}(m-\operatorname{del}(r))
$$

and by definition of $w^{\prime}(r)$ it holds

$$
w_{h}(M)=k \sum_{r \in S^{\prime}}(W+m-\operatorname{del}(r)+1)+\sum_{r \in R(L)}(m-\operatorname{del}(r)) .
$$

Hence

$$
w_{h}(M)=(W+m+1) k\left|S^{\prime}\right|-k \sum_{r \in S^{\prime}} \operatorname{del}(r)+\sum_{r \in R(L)} m-\sum_{r \in R(L)} \operatorname{del}(r) .
$$

By definition of feasible matching and by Lemma 18, $|V(T)|=|T|$. Furthermore, since $|T|=k\left|S^{\prime}\right|$, then $m k\left|S^{\prime}\right|=m|T|=m|V(T)|$. By construction $\sum_{r \in R(L)} m=m|V(L)|$ and $V(T) \cup V(L)=R_{\text {dist }}^{l} \cup R_{\text {safe }}^{l}$. Hence

$$
w_{h}(M)=(W+1) k\left|S^{\prime}\right|+m\left|R_{\text {dist }}^{l} \cup R_{\text {safe }}^{l}\right|-\left(k \sum_{r \in S^{\prime}} \operatorname{del}(r)+\sum_{r \in R(L)} \operatorname{del}(r)\right) .
$$




\section{Proof of Lemma 8}

Lemma 21. Let $R$ be an instance of $\langle|\Sigma|, m\rangle-A P$, let $\Pi_{S^{\prime}}$ be a feasible solution of $\langle|\Sigma|, m\rangle$-AP over instance $R$ that suppresses at most e entries, let $G_{R, S^{\prime}}$ be the graph associated with $R$ and $S^{\prime}$. Then there exists a complete matching of $G_{R, S^{\prime}}$ with total weight $w_{G}(M) \geq(W+1) k\left|S^{\prime}\right|+m\left|R_{\text {dist }}^{l} \cup R_{\text {safe }}^{l} \cup R_{\text {safe }}^{\prime l}\right|-e$.

Proof. Since $\Pi_{S^{\prime}}$ is feasible, we notice that each set of $\Pi_{S^{\prime}}$ associated with a resolution vector $r \in S^{\prime}$ must have cardinality at least $k$. Furthermore, we assume that all the sets of $\Pi_{S^{\prime}}$ are all associated with different resolution vectors, otherwise we can merge all the sets with the same resolution vector without increasing the cost of $\Pi_{S^{\prime}}$.

Let $x$ be a row of $S^{\prime}$ and denote by $R_{x} \subseteq R$ the set of rows of $R$ assigned to the set associated with resolution vector $x$. Starting from $\Pi_{S^{\prime}}$ we compute incrementally a matching $M$ by adding edges. First, for each set of vertices $T(x, i), 1 \leq i \leq k$, let $i^{*}$ be the minimum number such that $T\left(x, i^{*}\right)$ does not have any edge incident on it in $M$. First, assume that $x \in S_{\text {safe }}^{\prime}$; add the edge $\left(R_{\text {safe }}^{\prime l}(g, i), T(x, i)\right)$ to $M$, for each $1 \leq i \leq k$. Now, assume that $x \in S_{\text {cost }}^{\prime}$. Scan the rows in $R_{x}$ and for each row $r$ in $R_{x}$, if $r \in R_{\text {dist }}$ add the edge $\left(\bar{R}_{\text {dist }}^{l}(r), T\left(x, i^{*}\right)\right)$ to $M$. If $r \in R_{\text {safe }}$ and belongs to group $g$ add the edge $\left(R_{\text {safe }}^{l}(g, i), T\left(x, i^{*}\right)\right)$ to $M$. If no such $T\left(x, i^{*}\right)$ exists, then no edge is added to $M$. Notice that by construction, since all sets in $S^{\prime}$ have at least $k$ rows, then all vertices of $T$ are covered by $M$, therefore $M$ is feasible.

Finally add to $M$ all edges $\left(R_{\text {dist }}^{l}(x), R_{\text {dist }}^{r}(x)\right),\left(R_{\text {safe }}^{l}(g, i), R_{\text {safe }}^{r}(g, i)\right), 1 \leq i \leq \operatorname{exc}(g)$, for each vertex in $\left\{R_{\text {dist }}^{l}, R_{\text {safe }}^{l}\right\}$ respectively that is not already covered in $M$. Hence $M$ is complete.

Given a solution $\Pi_{S^{\prime}}$, a resolution vector $x$ of $S^{\prime}$ and the corresponding matching $M$, consider the order in which the rows of a set $R_{x}$ are scanned sequentially to construct $M$. Each of the first $k$ rows assigned to a cluster with resolution vector equal to $x$, by construction corresponds to an edge of $M$ joining a vertex of $V(T)$ and a vertex of $T$. Since $M$ is complete, those rows have a total cost in $\Pi_{S^{\prime}}$ of $k \sum_{r \in S^{\prime}} \operatorname{del}(r)$. The remaining rows of $R$ correspond to vertices of $V(L)$. Notice that those rows have a total cost in $\Pi_{S^{\prime}}$ not larger than $\sum_{r \in R(L)} \operatorname{del}(r)$. By Lemma $7 w_{h}(M)=(W+1) k\left|S^{\prime}\right|+m \mid R_{d i s t}^{l} \cup$ $R_{\text {safe }}^{l} \cup R_{\text {safe }}^{\prime l} \mid-\left(k \sum_{r \in S^{\prime}} \operatorname{del}(r)+\sum_{r \in R(L)} \operatorname{del}(r)\right)$. Since $\Pi_{S^{\prime}}$ suppresses at most $e$ entries of $R$, then $e \leq k \sum_{r \in S^{\prime}} \operatorname{del}(r)+\sum_{r \in R(L)} \operatorname{del}(r)$, therefore $w_{h}(M) \geq(W+1) k\left|S^{\prime}\right|+m\left|R_{\text {dist }}^{l} \cup R_{\text {safe }}^{l} \cup R_{\text {safe }}^{\prime l}\right|-e$.

\section{Proofs of Section 5}

It is easy to see that, by construction, the following properties hold.

Proposition 22. Let $r_{a}$, $r_{b}$ be two rows of $R_{i}$, with $r_{a}=g_{j}\left(v_{i}\right)$ and $r_{b}=g_{l}\left(v_{i}\right), j<l$. Let $r_{c}$ be a row of $R_{j}$, with $i \neq j$. Then:

- $H\left(r_{a}, r_{c}\right)=H\left(r_{b}, r_{c}\right)=3$;

- $H\left(r_{a}, r_{b}\right) \leq 2$;

- $H\left(r_{a}, r_{b}\right)=1$ iff $r_{a}=g_{h}\left(v_{i}\right)$ and $r_{b}=g_{h+3}\left(v_{i}\right)$, with $1 \leq h \leq 3$, or $r_{a}=g_{h}\left(v_{i}\right)$ and $r_{b}=g_{l}\left(v_{i}\right)$, with $4 \leq j \leq l \leq 6$.

Proposition 23. Let $r_{a}, r_{b}$ be two rows of $E_{i, j}$, with $r_{a} \in g_{h}\left(v_{i}, v_{j}\right)$ and $r_{b} \in g_{l}\left(v_{i}, v_{j}\right)$, with $h<l$. Let $r_{c}, r_{d}$ be two rows of $R_{i}$ and $R_{p}$, with $p \neq i, j$, and let $r_{e}$ be a row of $E_{t, z}$, with $t \neq i$ or $z \neq j$. Then:

- $H\left(r_{a}, r_{b}\right) \leq 2$;

- $H\left(r_{a}, r_{b}\right)=1$ iff $r_{a}=g_{h}\left(v_{i}, v_{j}\right)$ and $r_{b}=g_{h+1}\left(v_{i}, v_{j}\right)$, with $1 \leq h \leq 2$;

- $H\left(r_{a}, r_{c}\right)=1$ iff $r_{c}$ is in the docking group $d_{i, j}\left(g\left(v_{i}\right)\right)$ of $R_{i}$ and $r_{a}$ is in the group $g^{i}\left(v_{i}, v_{j}\right)$;

- $H\left(r_{a}, r_{c}\right)=2$ only if $r_{c}$ is in a group adjacent to $d_{i, j}\left(g\left(v_{i}\right)\right)$;

- $H\left(r_{a}, r_{d}\right)=3$;

- $H\left(r_{a}, r_{e}\right)=3$. 
In what follows, by an abuse of notation, we may use a group $g(\cdot)$ to denote its representative row $r_{g}(\cdot)$. Fig. 5 shows the groups of $R_{i}, R_{j}, E_{i, j}$. Each group of identical rows si represented with a vertex, while an edge joins two vertices iff the corresponding groups are at Hamming distance 1.

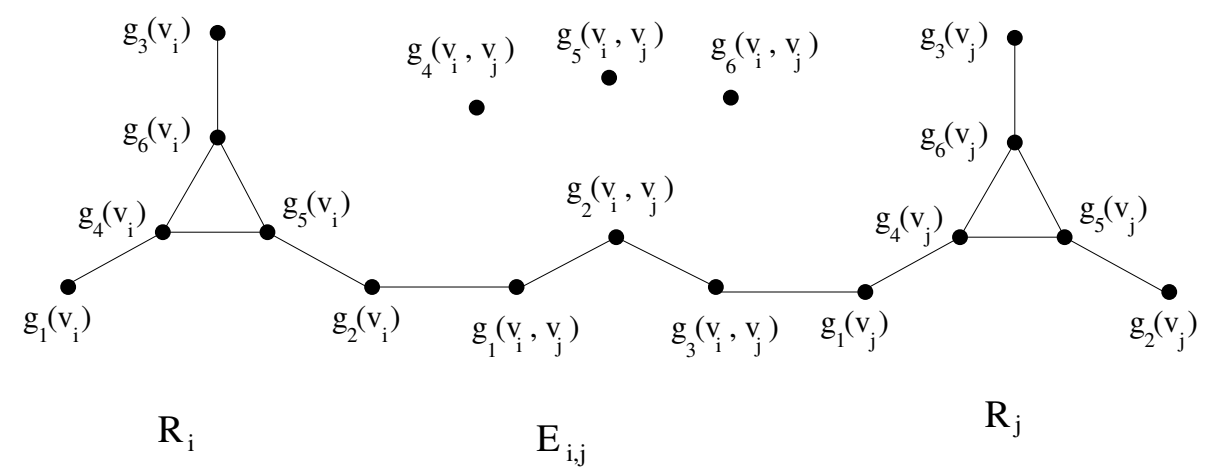

Figure 2: Groups at Hamming distance 1 in $R_{i}, R_{j}, E_{i, j}$ : vertices represent groups, while an edge joins two vertices representing groups at Hamming distance 1.

\section{Proof of Lemma 11}

Lemma 24. Let $R_{i}$ be a set of rows, then an optimal solution of $3-A P(3)$ over instance $R_{i}$ has a cost of at least 9 .

Proof. Let us consider the set of 9 rows, distributed in 6 groups, of $R_{i}$. As none of the group of $R_{i}$ consists of at least 3 rows, it follows that any solution of $3-\mathrm{AP}(3)$ suppresses at least one entry in each row of $R_{i}$, hence the lemma follows.

\section{Proof of Lemma 12}

In order to prove Lemma 12 first we have to show some properties of a canonical solution.

Lemma 25. Let $R_{i}$ be a set of rows. Then a solution of $3-A P(3)$ over instance $R$ induces an optimal cost for the set $R_{i}$ if it is a type a solution.

Proof. By construction, a type a solution is an optimal solution over instance $R_{i}$, as each row has a cost of 1 in a type a solution.

Now, in Lemma 26, we will prove a property of a type $b$ solution over the sets $R_{i}, E_{i, j}, E_{i, h}, E_{i, k}$.

Lemma 26. Let $S$ be a type b solution of 3-AP(3) over instance $R_{i} \cup E_{i, j} \cup E_{i, h} \cup E_{i, k}$, then $S$ suppresses 9 entries in the rows of $R_{i}$, and 1 entry for each row of $g^{i}\left(v_{i}, v_{j}\right), g^{i}\left(v_{i}, v_{h}\right), g^{i}\left(v_{i}, v_{k}\right)$.

Proof. By construction each set of a type $b$ solution containing a docking group of $R_{i}$ consists of three rows, where exactly one position is suppressed for each row by Prop. 23. The cluster $g_{4}\left(v_{i}\right) \cup g_{5}\left(v_{i}\right) \cup g_{6}\left(v_{i}\right)$ consists of 3 rows, where exactly one position is suppressed for each row. By a simple counting argument, the rows of $R_{i}$ have a total cost of 9 .

Let $\Pi$ be a solution of 3 - $\mathrm{AP}(3)$ over instance $R$ and let $E_{i, j}$ be an edge set. Then we say that $\Pi$ induce an $i$-normal solution for $E_{i, j}$ if it contains the following three sets: (i) one set clusters $C_{1}=g^{i}\left(v_{i}, v_{j}\right) \cup d_{i, j}\left(v_{i}\right)$, (ii) one set containing $C_{2} \supseteq \bigcup_{t \in\{4,5,6\}} g_{t}\left(v_{i}, v_{j}\right)$, such that exactly two entries (in columns 1 and 3 ) are suppressed in the rows of $C_{2}$, (iii) set $C_{3}=g_{2}\left(v_{i}, v_{j}\right) \cup g^{j}\left(v_{i}, v_{j}\right)$. 
Lemma 27. Let $\Pi$ be a solution of 3-AP(3) over instance $R$ and let $E_{i, j}$ be an edge set, then $\Pi$ suppresses at most 10 entries in the rows of $E_{i, j}$ only if it induces an $i$-normal or $j$-normal solution for $E_{i, j}$.

Proof. First assume that $\Pi$ induces an i-normal solution for $E_{i, j}$. By Prop. 23 , it follows that one entry of $g^{i}\left(v_{i}, v_{j}\right)$ is suppressed. In the set consisting of the rows in $g_{t}\left(v_{i}, v_{j}\right)$, with $t \in\{4,5,6\}$, by Prop. 23 two positions for each row are suppressed. Finally, by Prop. 23 in the set $g_{2}\left(v_{i}, v_{j}\right) \cup g^{j}\left(v_{i}, v_{j}\right)$, exactly one position is suppressed for each row.

Now, let us prove that if $\Pi$ is a solution that is not $\mathrm{i}$-normal or $\mathrm{j}$-normal for $E_{i, j}$, then $c_{\Pi}\left(E_{i, j}\right) \geq 11$. Notice that that each row in $g_{t}\left(v_{i}, v_{j}\right)$, with $t \in\{4,5,6\}$, has a Hamming distance 2 from any other row of $R \backslash g_{t}\left(v_{i}, v_{j}\right)$, hence at least two entries are suppressed in each solution П. Furthermore, notice that each of the four rows in the groups $g_{1}\left(v_{i}, v_{j}\right), g_{2}\left(v_{i}, v_{j}\right), g_{3}\left(v_{i}, v_{j}\right)$ must have a cost of at most 1 . But then, the rows of $g_{2}\left(v_{i}, v_{j}\right)$ must be co-clustered with the row of exactly one of $g_{1}\left(v_{i}, v_{j}\right), g_{3}\left(v_{i}, v_{j}\right)$ (w.l.o.g. $\left.g_{1}\left(v_{i}, v_{j}\right)\right)$. But then $g_{3}\left(v_{i}, v_{j}\right)=g^{i}\left(v_{i}, v_{j}\right)$, must be co-clustered with $d_{i, j}\left(v_{i}\right)$.

Lemma 28. Let $S$ be a solution of $3-A P(3)$ over instance $R$, then we can compute in polynomial time a solution $S^{\prime}$ such that $c\left(S^{\prime}\right) \leq c(S)$ and $S^{\prime}$ contains at most one set suppressing three entries for each row.

Proof. Assume that solution $S$ contains sets $Y_{1}, \ldots, Y_{p}$, with $p \geq 2$, such that all the positions of the rows in $Y_{j}$ are suppressed. Then we can compute in polynomial time a solution $S^{\prime}$ by merging the set $Y_{1}, \ldots, Y_{p}$ in a single cluster $Y$. Notice that $c\left(S^{\prime}\right) \leq c(S)$, as in both solution $S^{\prime}$ and $S$ three positions are suppressed for each row $r \in \bigcup_{j=1 \ldots p} Y_{j}$.

Now, let us first introduce some properties of a solution of 3-AP(3) over instance $R$.

Lemma 29. Let $\Pi$ be a solution of $3-A P(3)$ over instance $R$, we can compute in polynomial time a solution $\Pi^{\prime}$ such that

1. for each edge set $E_{i, j}$, $\Pi^{\prime}$ has a set $S_{i, j}$ containing the rows of groups $\bigcup_{t=4,5,6} g_{t}\left(v_{i}, v_{z}\right)$, such that for each row in $S_{i, j}$ exactly two columns (columns 1 and 3 ) are supprssed;

2. $c\left(\Pi^{\prime}\right) \leq c(\Pi)$.

Proof. First, notice that by Prop. 23 the rows in the groups $\cup_{t=4,5,6} g_{t}\left(v_{i}, v_{j}\right)$ have distance smaller than 3 only w.r.t. rows of $E_{i, j}$. Furthermore, notice that, by construction, each row in $\bigcup_{t=4,5,6} g_{t}\left(v_{i}, v_{j}\right)$ may be equal to another row of $R$ only in the second position.

Assume that there exist clusters $S_{1}, S_{2}, S_{3}$ (at most one of these clusters can be empty) containing the rows of $\bigcup_{t=4,5,6} g_{t}\left(v_{i}, v_{j}\right)$, such that at most two entries are suppressed for each row of the cluster $S_{j}$, $j \in\{1,2,3\}$. Then, for each row in $S_{1} \cup S_{2} \cup S_{3}$, the positions 1 and 3 are suppressed. Hence, we can merge clusters $S_{1}, S_{2}, S_{3}$, without increasing the cost of the solution, obtaining one set that contains the rows $\bigcup_{t=4,5,6} g_{t}\left(v_{i}, v_{z}\right)$.

Assume that some rows of $\cup_{t=4,5,6} g_{t}\left(v_{i}, v_{j}\right)$ are in the cluster $X$ and some rows of $\cup_{t=4,5,6} g_{t}\left(v_{i}, v_{j}\right)$ are in a different cluster $Y$, such that at most two entries are suppressed for each row of the cluster $Y$. Then we can move the rows of $\cup_{t=4,5,6} g_{t}\left(v_{i}, v_{j}\right) \cap X$ to $Y$, decreasing the cost of the solution.

Now, assume that these rows are all clustered in set $X$. It follows that each row of $\cup_{t=4,5,6} g_{t}\left(v_{i}, v_{j}\right)$ have a cost of 3 . Hence, we can move this set of rows to a new set $\cup_{t=4,5,6} g_{t}\left(v_{i}, v_{j}\right)$, decreasing the cost of the solution.

Lemma 30. Let $R_{i}, R_{j}$ be two set of rows and let $E_{i, j}$ be an edge set of $R$. Let $\Pi$ be a solution of 3-AP(3) over instance $R$ that associates a type $\mathrm{b}$ solution with both $R_{i}, R_{j}$. Then we can compute in polynomial time a solution $\Pi^{\prime}$ of $3-A P(3)$ over instance $R$ where exactly one of $R_{i}, R_{j}$ is associated with a type $\mathrm{b}$ solution and such that $c\left(\Pi^{\prime}\right) \leq c(\Pi)$.

Proof. Notice that, by Prop. 23, the rows of group $g_{2}\left(v_{i}, v_{j}\right)$ have Hamming distance 1 only from the rows of $g_{1}\left(v_{i}, v_{j}\right)$ and $g_{3}\left(v_{i}, v_{j}\right)$. Since in a type $b$ solution the rows of $g_{1}\left(v_{i}, v_{j}\right)$ and $g_{3}\left(v_{i}, v_{j}\right)$ are co-clustered with rows of $R_{i}$ and $R_{j}$, it follows by Prop. 23 that the rows of $g_{2}\left(v_{i}, v_{j}\right)$ are co-clustered in $\Pi$ with rows at 
Hamming distance at least 2. Hence, $\Pi$ suppresses two entries in each row of $g_{2}\left(v_{i}, v_{j}\right)$, and, as $g_{2}\left(v_{i}, v_{j}\right)$ consists of 2 rows, at least 4 entries of rows in $g_{2}\left(v_{i}, v_{j}\right)$ are suppressed in $\Pi$. Notice that, as $R_{i}$ and $R_{j}$ are associated with type $b$ solutions in $\Pi$, the only rows that can be clustered with the rows of $g_{2}\left(v_{i}, v_{j}\right)$ are those of groups $g_{4}\left(v_{i}, v_{j}\right), g_{5}\left(v_{i}, v_{j}\right), g_{6}\left(v_{i}, v_{j}\right)$.

Starting from solution $\Pi$, let us compute a solution $\Pi^{\prime}$ of 3 - $\mathrm{AP}(3)$ over instance $R$ as follows. Let $E_{i, j}, E_{i, t}, E_{i, z}$ be the edge sets associated with the three edges incident in $v_{i}$. Modify solution $\Pi$ so that $\Pi^{\prime}$ induces a type a solution for $R_{i}$, and a j-normal solution for $E_{i, j}$. Moreover, for each row of a group $g^{i}\left(v_{i}, v_{z}\right)$ of an edge set $E_{i, z}$, with $z \neq j$, co-cluster such group $g^{i}\left(v_{i}, v_{z}\right)$ with the cluster containing the rows of $\bigcup_{t=4,5,6} g_{t}\left(v_{i}, v_{z}\right)$.

By Lemma 27 the rows of edge set $E_{i, j}$ have a total cost of 10 . Notice that by Lemma 29, we can assume that $\Pi$ has a set $C$ containing $\bigcup_{t=4,5,6} g_{t}\left(v_{i}, v_{z}\right)$, such that exactly two entries (corresponding to the positions 1 and 3) are suppressed for each row in $C$. Hence the representative row of $C$ has Hamming distance 2 from $g^{i}\left(v_{i}, v_{z}\right)$, as they are equal in position 2 .

Now, each of these two rows $g^{i}\left(v_{i}, v_{z}\right)$ has a cost of 2 in $\Pi^{\prime}$, while it has a cost of least 1 in $\Pi$. Notice that each of the two rows of $g_{2}\left(v_{i}, v_{j}\right)$ has a cost of at least 2 in $\Pi$, while it has a cost of 1 in $\Pi^{\prime}$. Hence, $c\left(\Pi^{\prime}\right) \leq c(\Pi)$.

Lemma 31. Let $\Pi$ be a solution of the $3-A P(3)$ over instance $R$, such that two sets $R_{i}, R_{j}$ are not associated with a type b solution in $\Pi$ and $\Pi$ induces a total cost of 10 for the rows in $E_{i, j}$. Then at least one of $R_{i}, R_{j}$ has cost 11 .

Proof. Assume that $\Pi$ induces a total cost of 10 for the rows in $E_{i, j}$. Notice that the rows in $\bigcup_{t=4,5,6} g_{t}\left(v_{i}, v_{j}\right)$ have a total cost of 6 , as by Prop. 23 they are at Hamming distance at least 2 from any other row of $R$. Furthermore, the 4 rows of $E_{i, j} \backslash \bigcup_{t=4,5,6} g_{t}\left(v_{i}, v_{j}\right)$ must have a cost of at least 1 in $\Pi$. Notice that, by Lemma 27. $\Pi$ induces either an i-normal or j-normal solution for $E_{i, j}$ (w.l.o.g. we assume that is i-normal). Hence $g^{\imath}\left(v_{i}, v_{j}\right)$ is clustered with the rows of $\left.d_{i, j}\left(v_{i}\right)\right)$, while of $g^{j}\left(v_{i}, v_{j}\right)$ is clustered with $g_{2}\left(v_{i}, v_{j}\right)$, otherwise some rows of $g_{2}\left(v_{i}, v_{j}\right)$ are clustered in $\Pi$ with a row at Hamming distance at least 2 , hence the total cost of the rows in $E_{i, j}$ is greater than 10. But then, we claim that $\Pi$ induces a cost of at least 11 for the rows of the set $R_{i}$.

Now, recall that by hypothesis $R_{i}$ is not associated with a type $b$ solution in $\Pi$, and let us consider the clusters containing rows of $R_{i}$ in $\Pi$. Notice that if at least two rows of $R_{i}$ are clustered with some rows at Hamming distance at least 2 , then $\Pi$ induces a cost of at least 11 for the set $R_{i}$. Recall that group $d_{i, j}\left(g\left(v_{i}\right)\right)$ of $R_{i}$ is clustered only with rows of group $g^{i}\left(v_{i}, v_{j}\right)$ of $E_{i, j}$, and consider the cases that either the three groups of rows in $g_{4}\left(v_{i}\right), g_{5}\left(v_{i}\right), g_{6}\left(v_{i}\right)$ are co-clustered, or not. In the former case, as $R_{i}$ is not associated with a type $b$ solution, it follows that the rows of at least one of the docking group of $R_{i}$ are clustered with rows at Hamming distance 2 ; hence $\Pi$ induces a cost of at least 11 for the set $R_{i}$. In the latter case, let us consider the group of $R_{i}$ (w.l.o.g. $\left.g_{4}\left(v_{i}\right)\right)$ adjacent to $d_{i, j}\left(g\left(v_{i}\right)\right)$ and let $C$ be the cluster containing the unique row of $g_{4}\left(v_{i}\right)$. As the rows in $g_{4}\left(v_{i}\right), g_{5}\left(v_{i}\right), g_{6}\left(v_{i}\right)$ are not co-clustered, it follows that $C$ contains a row $r$ at Hamming distance at least 2 from $g_{4}\left(v_{i}\right)$. If $r \in R_{i}$, then $\Pi$ suppresses at least two entries of two rows of $R_{i}$, namely $r$ and $g_{4}\left(v_{i}\right)$, hence $\Pi$ induces a cost of at least 11 in rows of $R_{i}$. If $r \notin R_{i}$, then $r$ must be a row at Hamming distance 3 from $g_{4}\left(v_{i}\right)$. Indeed by Prop. 22 and by Prop. 23 , the rows at Hamming distance not greater than 2 from $g_{4}\left(v_{i}\right)$ belong to $R_{i} \cup\left(E_{i, j} \backslash \bigcup_{t=4,5,6} g_{t}\left(v_{i}, v_{j}\right)\right)$. We have assumed that $\left(R_{i} \backslash\left\{g_{4}\left(v_{i}\right)\right\}\right) \cap C=\emptyset$, and it must be $\left(E_{i, j} \backslash \bigcup_{t=4,5,6} g_{t}\left(v_{i}, v_{j}\right)\right) \cap C=\emptyset$, since by hypothesis $\Pi$ induces an i-normal solution for the rows in $E_{i, j}$. Hence $g_{4}\left(v_{i}\right)$ must have cost equal to 3 and must be part of the cluster $X$ in $\Pi$ by the Lemma 28 . It follows that $\Pi$ induces a cost of at least 11 for the set $R_{i}$.

Now, let us prove Lemma 12 .

Lemma 32. Let $\Pi$ be a solution of $3-A P(3)$ over instance $R$. Then we can compute in polynomial time a canonical solution $\Pi^{\prime}$ of $3-A P(3)$ over instance $R$ such that $c\left(\Pi^{\prime}\right) \leq c(\Pi)$. 
Proof. Let us consider the solution $\Pi$. Before computing a canonical solution $\Pi^{\prime}$, we compute an intermediate solution $\Pi^{\prime \prime}$ such that $c\left(\Pi^{\prime \prime}\right) \leq c(\Pi)$ as follows. First, for each set $R_{i}$, if $R_{i}$ is associated with a type $b$ solution in $\Pi$, then define a type $b$ solution for $R_{i}$ in $\Pi^{\prime \prime}$. Otherwise, if each docking vertices $d_{i, j}\left(v_{i}\right)$ of a set $R_{i}$ is clustered in $\Pi$ with the row of group $g^{i}\left(v_{i}, v_{j}\right)$ of $E_{i, j}$, then define a type $b$ solution for $R_{i}$ in $\Pi^{\prime \prime}$; else define a type a solution for $R_{i}$ in $\Pi^{\prime \prime}$. Furthermore, define a set containing row $x_{1}, x_{2}, x_{3}$ in $\Pi^{\prime \prime}$. Next, consider the rows of an edge set $E_{i, j}$, and define a clustering of the rows not yet clustered in $\Pi^{\prime \prime}$. If exactly one of $R_{i}, R_{j}$ (w.l.o.g. $R_{i}$ ) is associated with a type $b$ solution in $\Pi^{\prime \prime}$, then define an i-normal solution for $E_{i, j}$ in $\Pi^{\prime \prime}$. Else if at least one of $R_{i}, R_{j}$ (w.l.o.g. $R_{i}$ ) is associated with a type a solution in $\Pi^{\prime \prime}$, then define the following solution: one set contains the rows in $g^{i}\left(v_{i}, v_{j}\right) \cup g_{2}\left(v_{i}, v_{j}\right)$; one set contains the rows in $\bigcup_{t=4,5,6}\left(g_{t}\left(v_{i}, v_{j}\right)\right) \cup g^{j}\left(v_{i}, v_{j}\right)$. If both $R_{i}, R_{j}$ are associated with a type $b$ solution in $\Pi^{\prime \prime}$, then define a set $\bigcup_{t=4,5,6}\left(g_{t}\left(v_{i}, v_{j}\right)\right) \cup g_{2}\left(v_{i}, v_{j}\right)$ in $\Pi^{\prime \prime}$.

Now, let us show that $c(\Pi) \geq c\left(\Pi^{\prime \prime}\right)$. By Lemma 25 and by Lemma 26 it follows that for each row in a set $R_{i}$ the cost in $\Pi^{\prime \prime}$ is optimal. Furthermore, by Lemma 28, we can assume that $\Pi$ contains a set $X \supseteq\left\{x_{1}, x_{2}, x_{3}\right\}$, hence the rows in $\left\{x_{1}, x_{2}, x_{3}\right\}$ have all cost 3 in both $\Pi$ and $\Pi^{\prime \prime}$. Hence it remains to consider the cost of the edge set $E_{i, j}$.

Let $E_{i, j}$ be an edge set. Notice that by Lemma 29 we can assume that $\Pi$ contains a set $S_{i, j} \supseteq$ $\bigcup_{t=4,5,6} g_{t}\left(v_{i}, v_{j}\right)$, and by construction $\Pi^{\prime \prime}$ contains a set $S_{i, j}^{\prime} \supseteq \bigcup_{t=4,5,6} g_{t}\left(v_{i}, v_{j}\right)$. Hence each row of $g_{t}\left(v_{i}, v_{j}\right)$, with $t \in\{4,5,6\}$, has a cost equal to 2 in both $\Pi$, $\Pi^{\prime \prime}$. Let us consider the case when both sets $R_{i}$ and $R_{j}$ are associated with a type $b$ solution in both $\Pi$ and $\Pi^{\prime \prime}$. The groups of $E_{i, j}$ not co-clustered in a type $b$ solution of $R_{i}, R_{j}$, are $g_{2}\left(v_{i}, v_{j}\right), g_{4}\left(v_{i}, v_{j}\right), g_{5}\left(v_{i}, v_{j}\right), g_{6}\left(v_{i}, v_{j}\right)$. By construction, as the rows at Hamming distance 1 from $g_{2}\left(v_{i}, v_{j}\right)$ are clustered in the type $b$ solution of $R_{i}, R_{j}$ in $\Pi$ (hence cannot be co-clustered with $\left.g_{2}\left(v_{i}, v_{j}\right)\right)$, it follows that the rows $g_{2}\left(v_{i}, v_{j}\right)$ must be clustered with a row having Hamming distance at least 2 in $\Pi$. As $\Pi^{\prime \prime}$ contains the set $S_{i, j}^{\prime \prime}=\left(\bigcup_{t=4,5,6} g_{t}\left(v_{i}, v_{j}\right)\right) \cup g_{2}\left(v_{i}, v_{j}\right)$ and as $\Pi$ contains the set $S_{i, j} \supseteq \bigcup_{t=4,5,6} g_{t}\left(v_{i}, v_{j}\right)$, it follows that the cost of the rows in $E_{i, j}$ in solution $\Pi$ is greater or equal than the cost of the rows in $E_{i, j}$ in solution $\Pi^{\prime \prime}$.

Let us consider the case when exactly one of the sets $R_{i}$ and $R_{j}$ (w.l.o.g. $R_{j}$ ) is associated with a type $b$ solution in $\Pi^{\prime \prime}$ and in $\Pi$. By construction, $R_{i}$ is associated with a type a solution in $\Pi^{\prime \prime}$. By Lemma 27 it follows that that $c_{\Pi^{\prime \prime}}\left(E_{i, j}\right)=10$, and, as each row in $\bigcup_{t=4.5,6} g_{t}\left(v_{i}, v_{j}\right)$ has a cost of 2 in $\Pi^{\prime \prime}$, it follows that each row in $E_{i, j} \backslash\left(\bigcup_{t=4,5,6} g_{t}\left(v_{i}, v_{j}\right)\right)$ has a cost of 1 in $\Pi^{\prime \prime}$. As $\Pi$ contains the set $S_{i, j}$, it follows that $\Pi$ suppresses two entries in the rows of $\bigcup_{t=4,5,6} g_{t}\left(v_{i}, v_{j}\right)$, hence the cost of the rows in $E_{i, j}$ in solution $\Pi$ is greater or equal than the cost of the rows in $E_{i, j}$ in solution $\Pi^{\prime \prime}$.

Let us consider the case when at least one of the sets $R_{i}$ and $R_{j}$ (w.l.o.g. $R_{i}$ ) is associated with a type $b$ solution in $\Pi^{\prime \prime}$ and not in $\Pi$. Notice that by construction, the rows in groups $d_{i, j}\left(v_{i}\right), g^{i}\left(v_{j}, v_{j}\right)$ are clustered in both $\Pi$ and $\Pi^{\prime \prime}$. Now, if $\Pi$ induces a cost of at least 11 for the rows in $E_{i, j}$, since $\Pi^{\prime \prime}$ induces a cost of at most 11 for the rows in $E_{i, j}$ it follows that $c_{\Pi}\left(E_{i, j}\right) \geq c_{\Pi^{\prime \prime}}\left(E_{i, j}\right)$. If $\Pi$ induces a cost of 10 for the rows in $E_{i, j}$, then by Prop. $23 g_{2}\left(v_{i}, v_{j}\right)$ must be co-clustered with $g^{j}\left(v_{i}, v_{j}\right)$. Then, it follows that by construction $R_{j}$ is associated with a type a solution in $\Pi^{\prime \prime}$ and that the rows of $E_{i, j}$ have a total cost of 10 in $\Pi^{\prime \prime}$. Hence $c_{\Pi}\left(E_{i, j}\right) \geq c_{\Pi^{\prime \prime}}\left(E_{i, j}\right)$.

Now, let us consider the case when both $R_{i}, R_{j}$, are associated with a type a solution in $\Pi^{\prime \prime}$. In this case, by construction, the rows in the edge set $E_{i, j}$ have a total cost of 11 in $\Pi^{\prime \prime}$, while they have a cost of at least 10 in $\Pi$, as the rows in $\bigcup_{t=4,5,6} g_{t}\left(v_{i}, v_{j}\right)$ (contained in the set $S_{i, j}$ of $\Pi$ ) have a total cost of 6 , while each of the 4 rows of $E_{i, j} \backslash \bigcup_{t=4,5,6} g_{t}\left(v_{i}, v_{j}\right)$ has a cost of at least 1 in $\Pi$. Assume that the rows of $E_{i, j}$ have a total cost of 10 in $\Pi$. By Lemma 31. $\Pi$ induces a total cost of 11 for the rows of one of the sets $R_{i}, R_{j}$ (w.l.o.g. $R_{i}$ ). Notice that by Lemma $25 \Pi^{\prime \prime}$ induces a cost of 9 for all the set $R_{i}$. Now, let us consider the set $R_{i}$ and the three edge sets $E_{i, j}, E_{i, h}, E_{i, k}$. In what follows, we will consider the cost induced by $\Pi$ and by $\Pi^{\prime \prime}$ in the set $R_{i}$ and in some of the edge sets $E_{i, j}, E_{i, h}, E_{i, k}$. More precisely, for each edge set $E_{i, x}$ in $\left\{E_{i, j}, E_{i, h}, E_{i, k}\right\}$, let us consider its cost together with the cost of $R_{i}$ only if $d_{i, x}\left(v_{i}\right)$ and $g^{i}\left(v_{i}, v_{x}\right)$ are clustered in $\Pi$ (otherwise $E_{i, x}$ will be eventually be considered together with $R_{x}$ ). By construction the cost of at most two edge sets in $E_{i, j}, E_{i, h}, E_{i, k}$ (assume w.l.o.g. $E_{i, j}, E_{i, h}$ ) are considered together with the cost of $R_{i}$, otherwise $d_{i, x}\left(v_{i}\right)$ and $g^{i}\left(v_{i}, v_{x}\right)$ would be co-custered in $\Pi$, for each $x \in\{j, h, k\}$ and by construction $R_{i}$ would be associated with a type $b$ solution in $\Pi^{\prime \prime}$. Since $c_{\Pi}\left(E_{i, j}\right) \geq 10, c_{\Pi}\left(E_{i, h}\right) \geq 10, c_{\Pi}\left(R_{i}\right) \geq 11$, while $c_{\Pi^{\prime \prime}}\left(E_{i, j}\right)=11, c_{\Pi^{\prime \prime}}\left(E_{i, h}\right)=11$ and $c_{\Pi}\left(R_{i}\right)=9$ it follows 
that $c_{\Pi}\left(E_{i, j}\right)+c_{\Pi}\left(E_{i, h}\right)+c_{\Pi}\left(R_{i}\right) \geq c_{\Pi^{\prime \prime}}\left(E_{i, j}\right)+c_{\Pi^{\prime \prime}}\left(E_{i, h}\right)+c_{\Pi^{\prime \prime}}\left(R_{i}\right)$.

Now, we have shown that $c(\Pi) \geq c\left(\Pi^{\prime \prime}\right)$. Notice that $\Pi^{\prime \prime}$ may not be a canonical solution, as there may exist two sets $R_{i}, R_{j}$, with $E_{i, j}$ part of the instance, associated with a type $b$ solution in $\Pi^{\prime \prime}$. Now, applying Lemma 30 for each pair of sets $R_{i}, R_{j}$, associated with a type $b$ solution in $\Pi^{\prime \prime}$, with $E_{i, j}$ part of the instance, we can compute a canonical solution $\Pi^{\prime}$ such that $c\left(\Pi^{\prime \prime}\right) \geq c\left(\Pi^{\prime}\right)$. Hence $c(\Pi) \geq c\left(\Pi^{\prime}\right)$.

Lemma 33. Let $C$ be cover of $G$. Then, we can compute in polynomial time a solution $\Pi$ of $3-A P(3)$ over instance $R$ of cost $6|V|+3|C|+11|E|+9$.

Proof. We can define a solution $\Pi$ of $3-\mathrm{AP}(3)$ of cost $6|V|+3|C|+11|E|+9$, as follows. Define a type a solution for each $R_{i}$ associated with a vertex $v_{i} \in C$. Each of such sets has a cost of 9 .

Define a type $b$ solution for set $R_{i}$ associated with a vertex $v_{i} \in V-C$, and define an i-normal solution for the sets $E_{i, j}, E_{i, h}, E_{i, l}$. Each such set $R_{i}$ has a cost of 9 , and each edge set in $\left\{E_{i, j}, E_{i, h}, E_{i, l}\right\}$ has a cost of 10. Accounting this decreasing of the cost of the edge sets (from 11 to 10) to the set $R_{i}$, is equivalent to assign to a type $b$ solution a cost equal to 6 .

For any other edge set $E_{i, j}$ add to $\Pi$ the following sets: $S_{1}=g_{1}\left(v_{i}, v_{j}\right) \cup g_{2}\left(v_{i}, v_{j}\right), S_{2}=g_{3}\left(v_{i}, v_{j}\right) \cup$ $g_{4}\left(v_{i}, v_{j}\right) \cup g_{5}\left(v_{i}, v_{j}\right) \cup g_{6}\left(v_{i}, v_{j}\right)$. Each such edge set has a cost of 11. Finally, define a set $X=\left\{x_{1}, x_{2}, x_{3}\right\}$, having a total cost of 9 . 